\title{
A Computational Study on the Effect of Hydration on New Particle Formation in the Sulfuric Acid/Ammonia and Sulfuric Acid/Dimethylamine Systems - Supporting Information
}

\author{
Henning Henschel ${ }^{\mathrm{a}}$, Theo Kurtén ${ }^{\mathrm{b}}$, and Hanna Vehkamäki ${ }^{\mathrm{a}}$ \\ ${ }^{a}$ Division of Atmospheric Sciences, Department of Physics, P.O. Box 64, \\ 00014 University of Helsinki, Finland \\ ${ }^{\mathrm{b}}$ Laboratory of Physical Chemistry, Department of Chemistry, P.O. Box 55, \\ 00014 University of Helsinki, Finland
}

\section{Contents}

1 Gibbs Free Energies, Enthalpies, and Entropies of Cluster Formation S2

2 Stepwise Gibbs Free Hydration Energies \& Hydration Profiles

3 Collision Rates $\quad$ S6

4 Evaporation Rates $\quad$ S9

$\begin{array}{lll}5 & \text { Particle Formation Rates } & \text { S13 }\end{array}$

6 Impact of Collision Rate Enhancement $\quad$ S28

7 Cartesian Coordinates of Optimized Structures $\quad$ S31 


\section{Gibbs Free Energies, Enthalpies, and Entropies of Cluster Formation}

Table S1: Energies of cluster formation from monomers at $T=298.15 \mathrm{~K}$ and $p^{0}=1 \mathrm{~atm}$.

\begin{tabular}{|c|c|c|c|}
\hline Cluster & $\begin{array}{c}\Delta H^{0} \\
{\left[\mathrm{kcal} \mathrm{mol}^{-1}\right]} \\
\end{array}$ & $\begin{array}{c}\Delta G^{0} \\
{\left[\mathrm{kcal} \mathrm{mol}^{-1}\right]} \\
\end{array}$ & 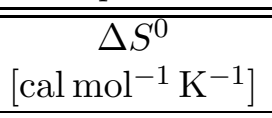 \\
\hline$\left(\mathrm{H}_{2} \mathrm{SO}_{4}\right)_{2}$ & -17.8487481487 & -7.8850671487 & -33.418352 \\
\hline$\left(\mathrm{H}_{2} \mathrm{SO}_{4}\right)_{3}$ & -35.8209843665 & -14.297199366 & -72.191130 \\
\hline$\left(\mathrm{H}_{2} \mathrm{SO}_{4}\right)_{4} \ldots$ & -52.6049004831 & -22.779974483 & -100.033294 \\
\hline $\mathrm{H}_{2} \mathrm{SO}_{4} \cdot \mathrm{H}_{2} \mathrm{O}$ & -11.4502372384 & -2.5972402384 & -29.693101 \\
\hline $\mathrm{H}_{2} \mathrm{SO}_{4} \cdot\left(\mathrm{H}_{2} \mathrm{O}\right)_{2}$ & -22.4899987472 & -4.3960777472 & -60.687311 \\
\hline $\mathrm{H}_{2} \mathrm{SO}_{4} \cdot\left(\mathrm{H}_{2} \mathrm{O}\right)_{3}$ & -33.4895124018 & -5.8342324018 & -92.756267 \\
\hline $\mathrm{H}_{2} \mathrm{SO}_{4} \cdot\left(\mathrm{H}_{2} \mathrm{O}\right)_{4}$ & -44.7799606822 & -7.0504936822 & -126.545254 \\
\hline $\mathrm{H}_{2} \mathrm{SO}_{4} \cdot\left(\mathrm{H}_{2} \mathrm{O}\right)_{5}$ & -54.5610576136 & -6.8079316136 & -160.164774 \\
\hline$\left(\mathrm{H}_{2} \mathrm{SO}_{4}\right)_{2} \cdot \mathrm{H}_{2} \mathrm{O}$ & -31.5924779044 & -11.2416769044 & -68.256920 \\
\hline$\left(\mathrm{H}_{2} \mathrm{SO}_{4}\right)_{2} \cdot\left(\mathrm{H}_{2} \mathrm{O}\right)_{2}$ & -43.8715146770 & -14.2645686770 & -99.302183 \\
\hline$\left(\mathrm{H}_{2} \mathrm{SO}_{4}\right)_{2} \cdot\left(\mathrm{H}_{2} \mathrm{O}\right)_{3}$ & -57.24365686008 & -16.49130886008 & -136.684046 \\
\hline$\left(\mathrm{H}_{2} \mathrm{SO}_{4}\right)_{2} \cdot\left(\mathrm{H}_{2} \mathrm{O}\right)_{4}$ & -71.5808181358 & -18.8567321358 & -176.837456 \\
\hline$\left(\mathrm{H}_{2} \mathrm{SO}_{4}\right)_{2} \cdot\left(\mathrm{H}_{2} \mathrm{O}\right)_{5}$ & -82.77652029746 & -20.62989629746 & -208.440803 \\
\hline$\left(\mathrm{H}_{2} \mathrm{SO}_{4}\right)_{3} \cdot \mathrm{H}_{2} \mathrm{O}$ & -52.29280998819 & -19.46748898819 & -110.096667 \\
\hline$\left(\mathrm{H}_{2} \mathrm{SO}_{4}\right)_{3} \cdot\left(\mathrm{H}_{2} \mathrm{O}\right)_{2}$ & -66.8265011609 & -22.5139491609 & -148.625030 \\
\hline$\left(\mathrm{H}_{2} \mathrm{SO}_{4}\right)_{3} \cdot\left(\mathrm{H}_{2} \mathrm{O}\right)_{3}$ & -80.9801138378 & -27.0430248378 & -180.905885 \\
\hline$\left(\mathrm{H}_{2} \mathrm{SO}_{4}\right)_{3} \cdot\left(\mathrm{H}_{2} \mathrm{O}\right)_{4}$ & -93.0780127967 & -30.4583267967 & -210.027458 \\
\hline$\left(\mathrm{H}_{2} \mathrm{SO}_{4}\right)_{3} \cdot\left(\mathrm{H}_{2} \mathrm{O}\right)_{5}$ & -103.6781696041 & -32.0805736041 & -240.139517 \\
\hline$\left(\mathrm{H}_{2} \mathrm{SO}_{4}\right)_{4} \cdot \mathrm{H}_{2} \mathrm{O}$ & -64.49520135434 & -25.45304135434 & -130.948047 \\
\hline$\left(\mathrm{H}_{2} \mathrm{SO}_{4}\right)_{4} \cdot\left(\mathrm{H}_{2} \mathrm{O}\right)_{2}$ & -87.6864501017 & -30.5773041017 & -191.545017 \\
\hline$\left(\mathrm{H}_{2} \mathrm{SO}_{4}\right)_{4} \cdot\left(\mathrm{H}_{2} \mathrm{O}\right)_{3}$ & -103.6440751327 & -36.7434651327 & -224.385750 \\
\hline$\left(\mathrm{H}_{2} \mathrm{SO}_{4}\right)_{4} \cdot\left(\mathrm{H}_{2} \mathrm{O}\right)_{4}$ & -111.9399020778 & -36.2690640778 & -253.801235 \\
\hline$\left(\mathrm{H}_{2} \mathrm{SO}_{4}\right)_{4} \cdot\left(\mathrm{H}_{2} \mathrm{O}\right)_{5}$ & -123.5102951737 & -38.5561431737 & -284.937624 \\
\hline $\mathrm{H}_{2} \mathrm{SO}_{4} \cdot \mathrm{NH}_{3}$ & -15.9984932548 & -7.60946625487 & -28.136935 \\
\hline$\left(\mathrm{H}_{2} \mathrm{SO}_{4}\right)_{2} \cdot \mathrm{NH}_{3}$ & -44.9962620190 & -23.8202770190 & -71.024601 \\
\hline$\left(\mathrm{H}_{2} \mathrm{SO}_{4}\right)_{2} \cdot\left(\mathrm{NH}_{3}\right)_{2}$ & -64.4605106074 & -33.3186676074 & -104.450256 \\
\hline$\left(\mathrm{H}_{2} \mathrm{SO}_{4}\right)_{3} \cdot \mathrm{NH}_{3}$ & -66.0568351924 & -33.9387921924 & -107.724444 \\
\hline$\left(\mathrm{H}_{2} \mathrm{SO}_{4}\right)_{3} \cdot\left(\mathrm{NH}_{3}\right)_{2}$ & -92.0891545072 & -49.4010455072 & -143.176618 \\
\hline$\left(\mathrm{H}_{2} \mathrm{SO}_{4}\right)_{3} \cdot\left(\mathrm{NH}_{3}\right)_{3}$ & -116.599033949 & -63.5327539495 & -177.985175 \\
\hline $\mathrm{NH}_{3} \cdot \mathrm{H}_{2} \mathrm{O}$ & -4.9369885788 & 0.3477644212 & -17.725152 \\
\hline $\mathrm{NH}_{3} \cdot\left(\overline{\mathrm{H}}_{2} \mathrm{O}\right)_{2}$ & -12.07985527073 & 4.80340972927 & -56.626749 \\
\hline $\mathrm{NH}_{3} \cdot\left(\mathrm{H}_{2} \mathrm{O}\right)_{3}$ & -21.45412795532 & 4.83025304468 & -88.158247 \\
\hline $\mathrm{NH}_{3} \cdot\left(\mathrm{H}_{2} \mathrm{O}\right)_{4} \ldots$ & -28.92742400040 & 5.80447399960 & -116.491359 \\
\hline $\mathrm{H}_{2} \mathrm{SO}_{4} \cdot \mathrm{NH}_{3} \cdot \mathrm{H}_{2} \mathrm{O}$ & -27.4130704248 & -9.2770774248 & -60.828419 \\
\hline $\mathrm{H}_{2} \mathrm{SO}_{4} \cdot \mathrm{NH}_{3} \cdot\left(\mathrm{H}_{2} \mathrm{O}\right)_{2}$ & -41.6472708222 & -13.5439088222 & -94.259139 \\
\hline $\mathrm{H}_{2} \mathrm{SO}_{4} \cdot \mathrm{NH}_{3} \cdot\left(\mathrm{H}_{2} \mathrm{O}\right)_{3}$ & -51.6657990767 & -14.7675070767 & -123.757481 \\
\hline
\end{tabular}




\begin{tabular}{|c|c|c|c|}
\hline Cluster & $\begin{array}{c}\Delta H^{0} \\
{\left[\mathrm{kcal} \mathrm{mol}^{-1}\right]}\end{array}$ & $\begin{array}{c}\Delta G^{0} \\
{\left[\mathrm{kcal} \mathrm{mol}^{-1}\right]}\end{array}$ & $\begin{array}{c}\Delta S^{0} \\
{\left[\mathrm{cal} \mathrm{mol}^{-1} \mathrm{~K}^{-1}\right]}\end{array}$ \\
\hline $\mathrm{H}_{2} \mathrm{SO}_{4} \cdot \mathrm{NH}_{3} \cdot\left(\mathrm{H}_{2} \mathrm{O}\right)_{4}$ & -61.3246841156 & -14.6968961156 & -156.390370 \\
\hline $\mathrm{H}_{2} \mathrm{SO}_{4} \cdot \mathrm{NH}_{3} \cdot\left(\mathrm{H}_{2} \mathrm{O}\right)_{5}$ & -72.3107816082 & -15.8345506082 & -189.422210 \\
\hline$\left(\mathrm{H}_{2} \mathrm{SO}_{4}\right)_{2} \cdot \mathrm{NH}_{3} \cdot \mathrm{H}_{2} \mathrm{O}$ & -57.4082981406 & -26.4986911406 & -103.671333 \\
\hline$\left(\mathrm{H}_{2} \mathrm{SO}_{4}\right)_{2} \cdot \mathrm{NH}_{3} \cdot\left(\mathrm{H}_{2} \mathrm{O}\right)_{2}$ & -68.4908608729 & -27.8183968729 & -136.416115 \\
\hline$\left(\mathrm{H}_{2} \mathrm{SO}_{4}\right)_{2} \cdot \mathrm{NH}_{3} \cdot\left(\mathrm{H}_{2} \mathrm{O}\right)_{3}$ & -83.4867125594 & -30.4897325594 & -177.752744 \\
\hline$\left(\mathrm{H}_{2} \mathrm{SO}_{4}\right)_{2} \cdot \mathrm{NH}_{3} \cdot\left(\mathrm{H}_{2} \mathrm{O}\right)_{4}$ & -94.2166031902 & -31.1513431902 & -211.521922 \\
\hline$\left(\mathrm{H}_{2} \mathrm{SO}_{4}\right)_{2} \cdot \mathrm{NH}_{3} \cdot\left(\mathrm{H}_{2} \mathrm{O}\right)_{5}$ & -103.3126684341 & -32.2977364341 & -238.185253 \\
\hline$\left(\mathrm{H}_{2} \mathrm{SO}_{4}\right)_{2} \cdot\left(\mathrm{NH}_{3}\right)_{2} \cdot \mathrm{H}_{2} \mathrm{O}$ & -78.8230893604 & -37.4565883604 & -138.743922 \\
\hline$\left(\mathrm{H}_{2} \mathrm{SO}_{4}\right)_{2} \cdot\left(\mathrm{NH}_{3}\right)_{2} \cdot\left(\mathrm{H}_{2} \mathrm{O}\right)_{2}$ & -93.0550913448 & -40.8781103448 & -175.002453 \\
\hline$\left(\mathrm{H}_{2} \mathrm{SO}_{4}\right)_{2} \cdot\left(\mathrm{NH}_{3}\right)_{2} \cdot\left(\mathrm{H}_{2} \mathrm{O}\right)_{3}$ & -102.8117445132 & -41.0718475132 & -207.076630 \\
\hline$\left(\mathrm{H}_{2} \mathrm{SO}_{4}\right)_{2} \cdot\left(\mathrm{NH}_{3}\right)_{2} \cdot\left(\mathrm{H}_{2} \mathrm{O}\right)_{4}$ & -112.1966825436 & -41.5025925436 & -237.109143 \\
\hline$\left(\mathrm{H}_{2} \mathrm{SO}_{4}\right)_{2} \cdot\left(\mathrm{NH}_{3}\right)_{2} \cdot\left(\mathrm{H}_{2} \mathrm{O}\right)_{5}$ & -123.4477509296 & -42.8975519296 & -270.166694 \\
\hline$\left(\mathrm{H}_{2} \mathrm{SO}_{4}\right)_{3} \cdot \mathrm{NH}_{3} \cdot \mathrm{H}_{2} \mathrm{O}$ & -80.6130345276 & -37.1982755276 & -145.613816 \\
\hline$\left(\mathrm{H}_{2} \mathrm{SO}_{4}\right)_{3} \cdot \mathrm{NH}_{3} \cdot\left(\mathrm{H}_{2} \mathrm{O}\right)_{2}$ & -93.6868102795 & -40.6347922795 & -177.937339 \\
\hline$\left(\mathrm{H}_{2} \mathrm{SO}_{4}\right)_{3} \cdot \mathrm{NH}_{3} \cdot\left(\mathrm{H}_{2} \mathrm{O}\right)_{3}$ & -105.5330540499 & -43.0164400499 & -209.681754 \\
\hline$\left(\mathrm{H}_{2} \mathrm{SO}_{4}\right)_{3} \cdot \mathrm{NH}_{3} \cdot\left(\mathrm{H}_{2} \mathrm{O}\right)_{4}$ & -116.1290931284 & -43.4153231284 & -243.883183 \\
\hline$\left(\mathrm{H}_{2} \mathrm{SO}_{4}\right)_{3} \cdot \mathrm{NH}_{3} \cdot\left(\mathrm{H}_{2} \mathrm{O}\right)_{5}$ & -128.0979251771 & -44.8539241771 & -279.201752 \\
\hline$\left(\mathrm{H}_{2} \mathrm{SO}_{4}\right)_{3} \cdot\left(\mathrm{NH}_{3}\right)_{2} \cdot \mathrm{H}_{2} \mathrm{O}$ & -107.2671904784 & -52.6973984784 & -183.027981 \\
\hline$\left(\mathrm{H}_{2} \mathrm{SO}_{4}\right)_{3} \cdot\left(\mathrm{NH}_{3}\right)_{2} \cdot\left(\mathrm{H}_{2} \mathrm{O}\right)_{2}$ & -116.4617828953 & -54.5960028953 & -207.498846 \\
\hline$\left(\mathrm{H}_{2} \mathrm{SO}_{4}\right)_{3} \cdot\left(\mathrm{NH}_{3}\right)_{2} \cdot\left(\mathrm{H}_{2} \mathrm{O}\right)_{3}$ & -128.3266447353 & -56.5570007353 & -240.716569 \\
\hline$\left(\mathrm{H}_{2} \mathrm{SO}_{4}\right)_{3} \cdot\left(\mathrm{NH}_{3}\right)_{2} \cdot\left(\mathrm{H}_{2} \mathrm{O}\right)_{4}$ & -141.5674231946 & -57.1564081946 & -283.115935 \\
\hline$\left(\mathrm{H}_{2} \mathrm{SO}_{4}\right)_{3} \cdot\left(\mathrm{NH}_{3}\right)_{2} \cdot\left(\mathrm{H}_{2} \mathrm{O}\right)_{5}$ & -152.7696105902 & -58.8650135902 & -314.957567 \\
\hline$\left(\mathrm{H}_{2} \mathrm{SO}_{4}\right)_{3} \cdot\left(\mathrm{NH}_{3}\right)_{3} \cdot \mathrm{H}_{2} \mathrm{O}$ & -126.7523186512 & -64.9988776512 & -207.122059 \\
\hline$\left(\mathrm{H}_{2} \mathrm{SO}_{4}\right)_{3} \cdot\left(\mathrm{NH}_{3}\right)_{3} \cdot\left(\mathrm{H}_{2} \mathrm{O}\right)_{2}$ & -139.3956910160 & -67.0251390160 & -242.732023 \\
\hline$\left(\mathrm{H}_{2} \mathrm{SO}_{4}\right)_{3} \cdot\left(\mathrm{NH}_{3}\right)_{3} \cdot\left(\mathrm{H}_{2} \mathrm{O}\right)_{3}$ & -147.9362481635 & -66.8922321635 & -271.822963 \\
\hline$\left(\mathrm{H}_{2} \mathrm{SO}_{4}\right)_{3} \cdot\left(\mathrm{NH}_{3}\right)_{3} \cdot\left(\mathrm{H}_{2} \mathrm{O}\right)_{4}$ & -162.9112451186 & -69.3759851186 & -313.718803 \\
\hline $\mathrm{H}_{2} \mathrm{SO}_{4} \cdot \mathrm{DMA}$ & -24.6465441592 & -15.4013431592 & -31.008553 \\
\hline$\left(\mathrm{H}_{2} \mathrm{SO}_{4}\right)_{2} \cdot \mathrm{DMA}$ & -57.0595364880 & -34.8753244880 & -74.406210 \\
\hline$\left(\mathrm{H}_{2} \mathrm{SO}_{4}\right)_{2} \cdot(\mathrm{DMA})_{2}$ & -87.5692739780 & -54.2563479780 & -111.732097 \\
\hline$\left(\mathrm{H}_{2} \mathrm{SO}_{4}\right)_{3} \cdot \mathrm{DMA}$ & -81.4604213712 & -47.7996073712 & -112.898920 \\
\hline$\left(\mathrm{H}_{2} \mathrm{SO}_{4}\right)_{3} \cdot(\mathrm{DMA})_{2}$ & -113.5835639644 & -67.2584459644 & -155.375202 \\
\hline$\left(\mathrm{H}_{2} \mathrm{SO}_{4}\right)_{3} \cdot(\mathrm{DMA})_{3}$ & -146.5927558198 & -89.9420848198 & -190.007277 \\
\hline $\mathrm{DM} A \mathrm{H}_{2} \mathrm{O}$ & -6.2023181114 & 1.9239968886 & -27.255790 \\
\hline $\mathrm{H}_{2} \mathrm{SO}_{4} \cdot \mathrm{DMA}^{\prime} \cdot \mathrm{H}_{2} \mathrm{O}$ & -37.8943672268 & -18.2871092268 & -65.763065 \\
\hline $\mathrm{H}_{2} \mathrm{SO}_{4} \cdot \mathrm{DMA} \cdot\left(\mathrm{H}_{2} \mathrm{O}\right)_{2}$ & -50.1106517255 & -20.6587217255 & -98.782257 \\
\hline $\mathrm{H}_{2} \mathrm{SO}_{4} \cdot \mathrm{DMA} \cdot\left(\mathrm{H}_{2} \mathrm{O}\right)_{3}$ & -60.4038733789 & -20.4171063789 & -134.116273 \\
\hline $\mathrm{H}_{2} \mathrm{SO}_{4} \cdot \mathrm{DMA} \cdot\left(\mathrm{H}_{2} \mathrm{O}\right)_{4}$ & -70.7771168473 & -21.2129478473 & -166.239038 \\
\hline $\mathrm{H}_{2} \mathrm{SO}_{4} \cdot \mathrm{DMA} \cdot\left(\mathrm{H}_{2} \mathrm{O}\right)_{5}$ & -80.6154010883 & -21.1290500883 & -199.518199 \\
\hline$\left(\mathrm{H}_{2} \mathrm{SO}_{4}\right)_{2} \cdot \mathrm{DMA} \cdot \mathrm{H}_{2} \mathrm{O}$ & -68.9625206960 & -37.3493056960 & -106.031240 \\
\hline$\left(\mathrm{H}_{2} \mathrm{SO}_{4}\right)_{2} \cdot \mathrm{DMA} \cdot\left(\mathrm{H}_{2} \mathrm{O}\right)_{2}$ & -78.6276362512 & -37.7357562512 & -137.152038 \\
\hline$\left(\mathrm{H}_{2} \mathrm{SO}_{4}\right)_{2} \cdot \mathrm{DMA} \cdot\left(\mathrm{H}_{2} \mathrm{O}\right)_{3}$ & -93.14387624224 & -40.5652902422 & -176.349443 \\
\hline
\end{tabular}




\begin{tabular}{|c|c|c|c|}
\hline Cluster & $\begin{array}{c}\Delta H^{0} \\
{\left[\mathrm{kcal} \mathrm{mol}^{-1}\right]}\end{array}$ & $\begin{array}{c}\Delta G^{0} \\
{\left[\mathrm{kcal} \mathrm{mol}^{-1}\right]}\end{array}$ & $\begin{array}{c}\Delta S^{0} \\
{\left[\mathrm{cal} \mathrm{mol}{ }^{-1} \mathrm{~K}^{-1}\right]}\end{array}$ \\
\hline$\left(\mathrm{H}_{2} \mathrm{SO}_{4}\right)_{2} \cdot \mathrm{DMA} \cdot\left(\mathrm{H}_{2} \mathrm{O}\right)_{4}$ & -104.3513591905 & -41.1348271905 & -212.029289 \\
\hline$\left(\mathrm{H}_{2} \mathrm{SO}_{4}\right)_{2} \cdot \mathrm{DMA} \cdot\left(\mathrm{H}_{2} \mathrm{O}\right)_{5}$ & -112.8329322089 & -40.8954722089 & -241.279424 \\
\hline$\left(\mathrm{H}_{2} \mathrm{SO}_{4}\right)_{2} \cdot(\mathrm{DMA})_{2} \cdot \mathrm{H}_{2} \mathrm{O}$ & -96.6584373642 & -52.9029113642 & -146.756751 \\
\hline$\left(\mathrm{H}_{2} \mathrm{SO}_{4}\right)_{2} \cdot(\mathrm{DMA})_{2} \cdot\left(\mathrm{H}_{2} \mathrm{O}\right)_{2}$ & -107.1165503052 & -54.7635333052 & -175.592878 \\
\hline$\left(\mathrm{H}_{2} \mathrm{SO}_{4}\right)_{2} \cdot(\mathrm{DMA})_{2} \cdot\left(\mathrm{H}_{2} \mathrm{O}\right)_{3}$ & -116.6018181841 & -53.6321361841 & -211.201348 \\
\hline$\left(\mathrm{H}_{2} \mathrm{SO}_{4}\right)_{2} \cdot(\mathrm{DMA})_{2} \cdot\left(\mathrm{H}_{2} \mathrm{O}\right)_{4}$ & -130.0477157071 & -55.5919987071 & -249.725700 \\
\hline$\left(\mathrm{H}_{2} \mathrm{SO}_{4}\right)_{2} \cdot(\mathrm{DMA})_{2} \cdot\left(\mathrm{H}_{2} \mathrm{O}\right)_{5}$ & -138.0301267860 & -56.0308097860 & -275.027057 \\
\hline$\left(\mathrm{H}_{2} \mathrm{SO}_{4}\right)_{3} \cdot \mathrm{DMA}^{-} \mathrm{H}_{2} \mathrm{O}$ & -91.4084921947 & $-47.7 \overline{4} 30411947$ & -146.454641 \\
\hline$\left(\mathrm{H}_{2} \mathrm{SO}_{4}\right)_{3} \cdot \mathrm{DMA} \cdot\left(\mathrm{H}_{2} \mathrm{O}\right)_{2}$ & -106.2605276559 & -51.9644666559 & -182.109883 \\
\hline$\left(\mathrm{H}_{2} \mathrm{SO}_{4}\right)_{3} \cdot \mathrm{DMA} \cdot\left(\mathrm{H}_{2} \mathrm{O}\right)_{3}$ & -117.2165296153 & -52.6906916153 & -216.420721 \\
\hline$\left(\mathrm{H}_{2} \mathrm{SO}_{4}\right)_{3} \cdot \mathrm{DMA} \cdot\left(\mathrm{H}_{2} \mathrm{O}\right)_{4}$ & -128.1871879283 & -53.8550939283 & -249.311068 \\
\hline$\left(\mathrm{H}_{2} \mathrm{SO}_{4}\right)_{3} \cdot \mathrm{DMA} \cdot\left(\mathrm{H}_{2} \mathrm{O}\right)_{5}$ & -137.2639506854 & -54.3753996854 & -278.009566 \\
\hline$\left(\mathrm{H}_{2} \mathrm{SO}_{4}\right)_{3} \cdot(\overline{\mathrm{DMA}})_{2} \cdot \mathrm{H}_{2} \mathrm{O}$ & -124.8550573196 & -69.0949853196 & -187.020195 \\
\hline$\left(\mathrm{H}_{2} \mathrm{SO}_{4}\right)_{3} \cdot(\mathrm{DMA})_{2} \cdot\left(\mathrm{H}_{2} \mathrm{O}\right)_{2}$ & -136.7100467207 & -71.2276487207 & -219.629039 \\
\hline$\left(\mathrm{H}_{2} \mathrm{SO}_{4}\right)_{3} \cdot(\mathrm{DMA})_{2} \cdot\left(\mathrm{H}_{2} \mathrm{O}\right)_{3}$ & -147.8286481914 & -70.5701501914 & -259.126272 \\
\hline$\left(\mathrm{H}_{2} \mathrm{SO}_{4}\right)_{3} \cdot(\mathrm{DMA})_{2} \cdot\left(\mathrm{H}_{2} \mathrm{O}\right)_{4}$ & -163.2477004790 & -75.4515514790 & -294.469725 \\
\hline$\left(\mathrm{H}_{2} \mathrm{SO}_{4}\right)_{3} \cdot(\mathrm{DMA})_{2} \cdot\left(\mathrm{H}_{2} \mathrm{O}\right)_{5}$ & -172.6993348634 & -77.0874378634 & -320.683876 \\
\hline$\left(\mathrm{H}_{2} \mathrm{SO}_{4}\right)_{3} \cdot(\mathrm{DMA})_{3} \cdot \mathrm{H}_{2} \mathrm{O}$ & -156.8557647344 & -89.8712607344 & -224.667123 \\
\hline$\left(\mathrm{H}_{2} \mathrm{SO}_{4}\right)_{3} \cdot(\mathrm{DMA})_{3} \cdot\left(\mathrm{H}_{2} \mathrm{O}\right)_{2}$ & -171.0448681086 & -92.8541381086 & -262.252990 \\
\hline$\left(\mathrm{H}_{2} \mathrm{SO}_{4}\right)_{3} \cdot(\mathrm{DMA})_{3} \cdot\left(\mathrm{H}_{2} \mathrm{O}\right)_{3}$ & -176.9414568719 & -91.5114328719 & -286.533702 \\
\hline$\left(\mathrm{H}_{2} \mathrm{SO}_{4}\right)_{3} \cdot(\mathrm{DMA})_{3} \cdot\left(\mathrm{H}_{2} \mathrm{O}\right)_{4}$ & -192.7576700101 & -93.6444850101 & -332.427250 \\
\hline
\end{tabular}




\section{Stepwise Gibbs Free Hydration Energies \& Hydration Profiles}

Table S2: Stepwise Gibbs free energies of hydration at $T=298.15 \mathrm{~K}$ and $p^{0}=1 \mathrm{~atm}$ $\left(\Delta_{1} \Delta_{\text {hydr }} G^{0}\right)$ in kcal mol${ }^{-1}$.

\begin{tabular}{lcccc}
\hline \hline \multirow{2}{*}{ Cluster } & \multicolumn{4}{c}{$\mathrm{n}\left(\mathrm{H}_{2} \mathrm{O}\right)$} \\
\cline { 2 - 5 } & 1 & 2 & 3 & 4 \\
\hline$\left(\mathrm{H}_{2} \mathrm{SO}_{4}\right)_{3} \cdot\left(\mathrm{NH}_{3}\right)_{3}$ & -1.47 & -2.02 & 0.13 & -2.48 \\
$\left(\mathrm{H}_{2} \mathrm{SO}_{4}\right)_{3} \cdot(\mathrm{DMA})_{3}$ & 0.07 & -2.98 & 1.34 & -2.13 \\
\hline \hline
\end{tabular}

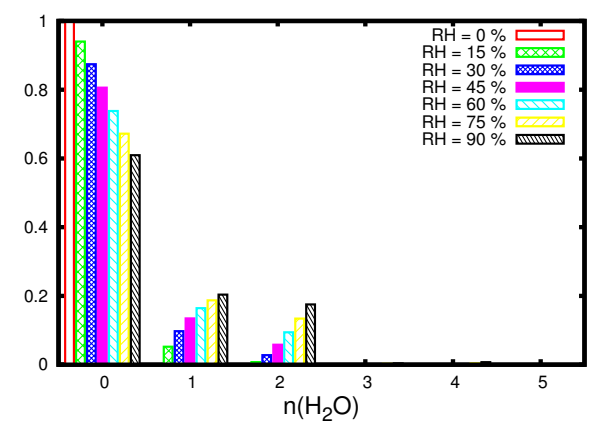

Figure S1: Hydrate populations for $\left(\mathrm{H}_{2} \mathrm{SO}_{4}\right)_{3} \cdot\left(\mathrm{NH}_{3}\right)_{3}$.

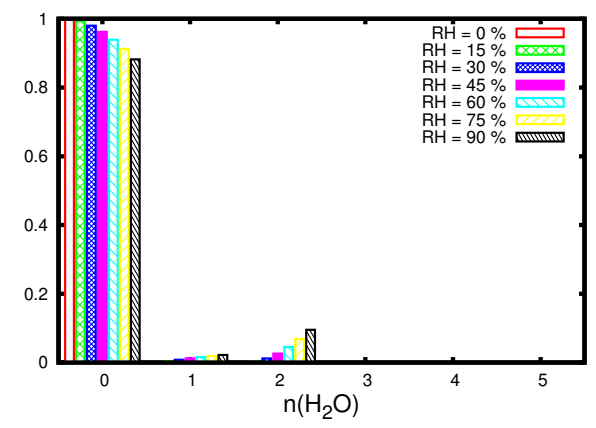

Figure S2: Hydrate populations for $\left(\mathrm{H}_{2} \mathrm{SO}_{4}\right)_{3} \cdot(\mathrm{DMA})_{3}$. 


\section{Collision Rates}

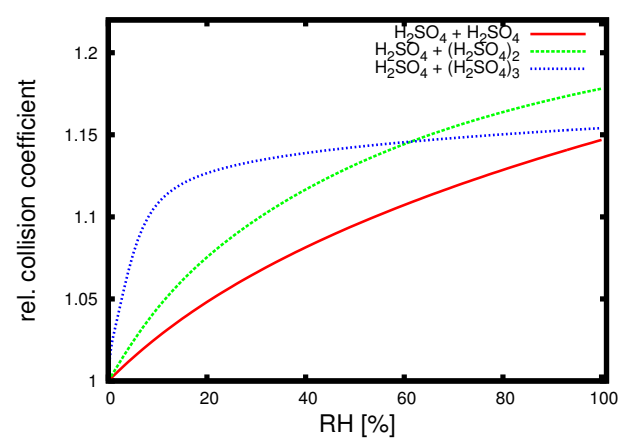

Figure S3: Relative collision coefficients for $\left(\mathrm{H}_{2} \mathrm{SO}_{4}\right)_{1}$ with $\left(\mathrm{H}_{2} \mathrm{SO}_{4}\right)_{\mathrm{x}}$.

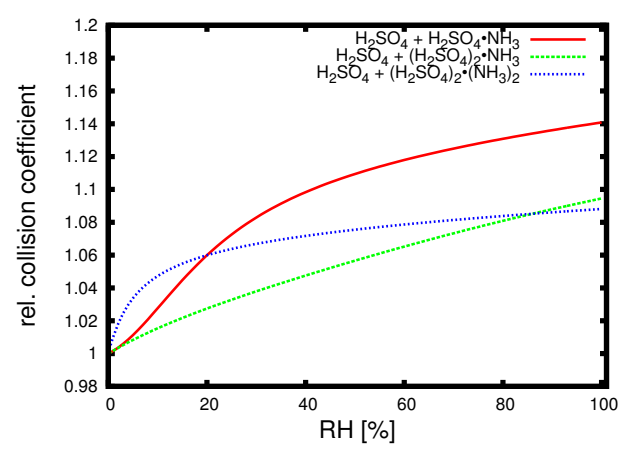

Figure S4: Relative collision coefficients for $\left(\mathrm{H}_{2} \mathrm{SO}_{4}\right)_{1}$ with $\left(\mathrm{H}_{2} \mathrm{SO}_{4}\right)_{\mathrm{x}} \cdot\left(\mathrm{NH}_{3}\right)_{\mathrm{y}}$.

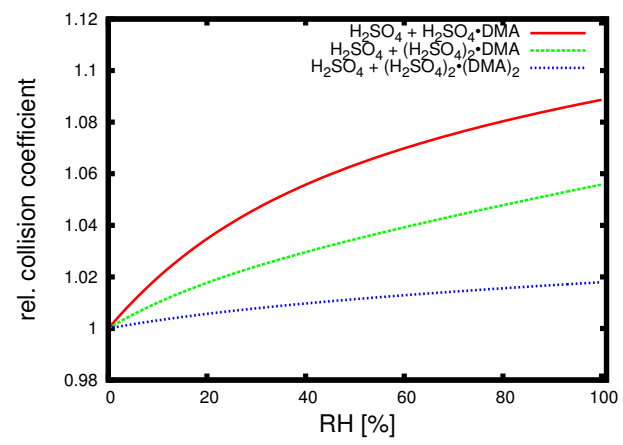

Figure S5: Relative collision coefficients for $\left(\mathrm{H}_{2} \mathrm{SO}_{4}\right)_{1}$ with $\left(\mathrm{H}_{2} \mathrm{SO}_{4}\right)_{\mathrm{x}} \cdot(\mathrm{DMA})_{\mathrm{y}}$. 


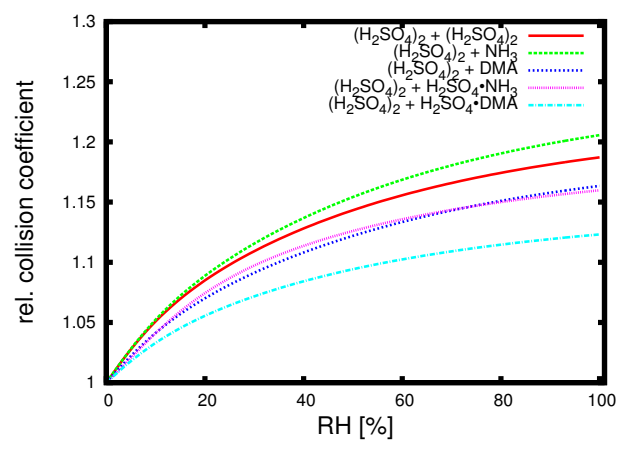

Figure S6: Relative collision coefficients for $\left(\mathrm{H}_{2} \mathrm{SO}_{4}\right)_{2}$.

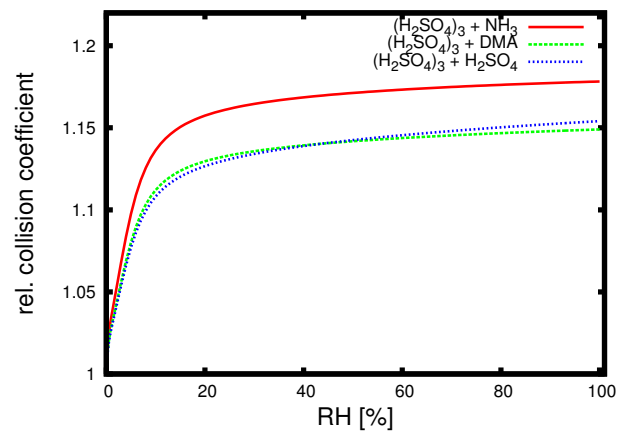

Figure S7: Relative collision coefficients for $\left(\mathrm{H}_{2} \mathrm{SO}_{4}\right)_{3}$.

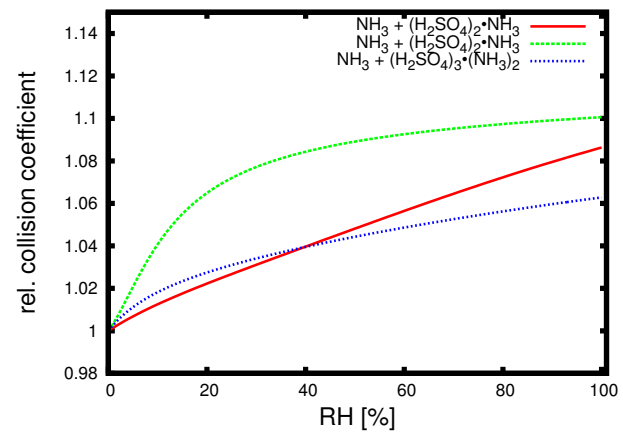

Figure S8: Relative collision coefficients for $\left(\mathrm{NH}_{3}\right)_{1}$. 


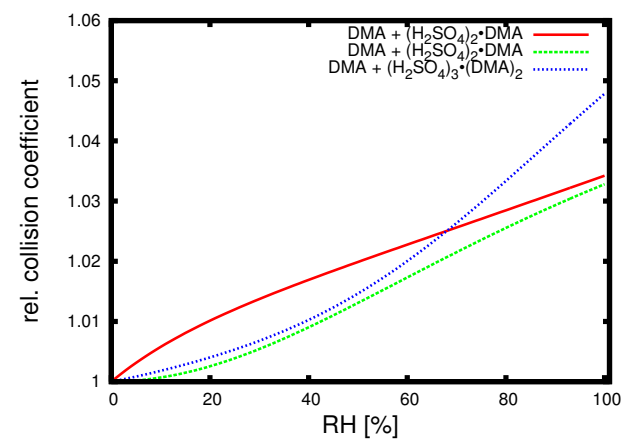

Figure S9: Relative collision coefficients for (DMA) $)_{1}$.

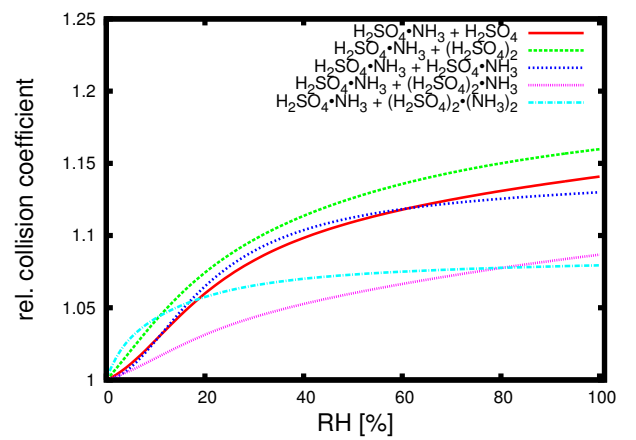

Figure S10: Relative collision coefficients for $\left(\mathrm{H}_{2} \mathrm{SO}_{4}\right)_{3} \cdot\left(\mathrm{NH}_{3}\right)$.

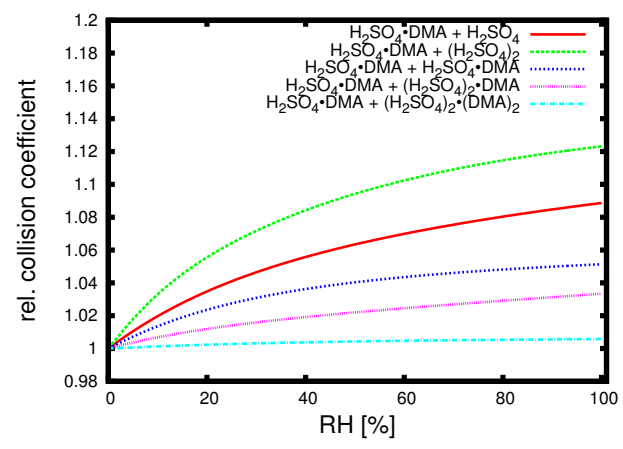

Figure S11: Relative collision coefficients for $\left(\mathrm{H}_{2} \mathrm{SO}_{4}\right)_{1} \cdot$ DMA. 


\section{Evaporation Rates}

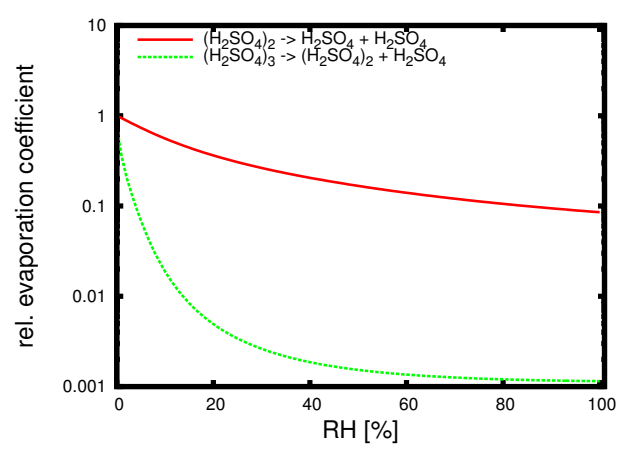

Figure S12: Relative evaporation coefficients for $\left(\mathrm{H}_{2} \mathrm{SO}_{4}\right)_{2,3}$.

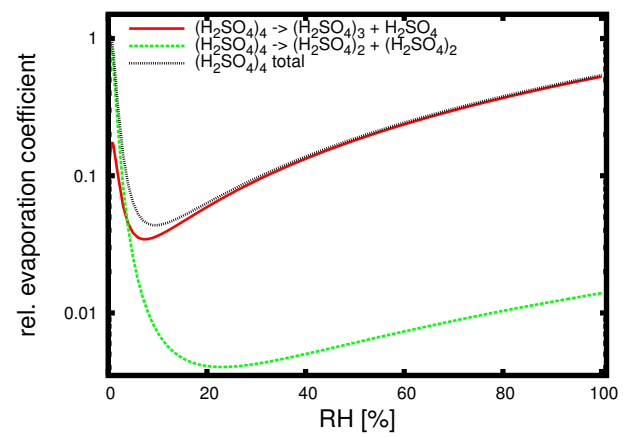

Figure S13: Relative evaporation coefficients for $\left(\mathrm{H}_{2} \mathrm{SO}_{4}\right)_{4}$.

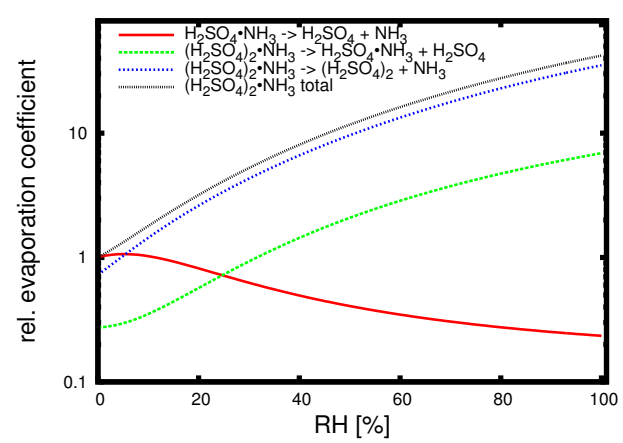

Figure S14: Relative evaporation coefficients for $\left(\mathrm{H}_{2} \mathrm{SO}_{4}\right)_{1,2} \cdot \mathrm{NH}_{3}$. 


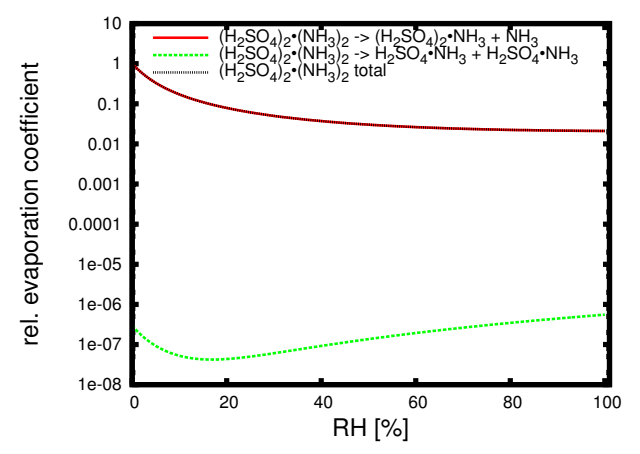

Figure S15: Relative evaporation coefficients for $\left(\mathrm{H}_{2} \mathrm{SO}_{4}\right)_{2} \cdot\left(\mathrm{NH}_{3}\right)_{2}$.

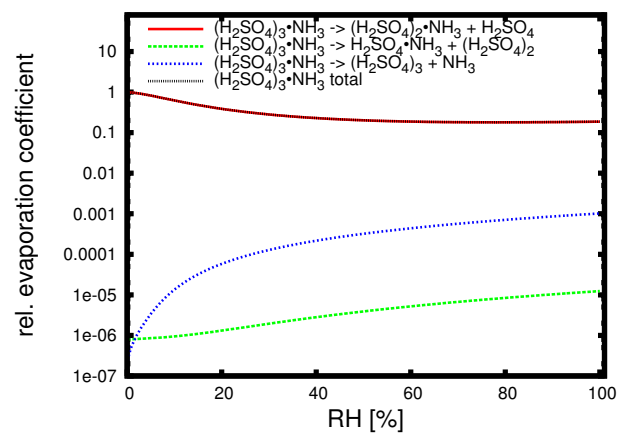

Figure S16: Relative evaporation coefficients for $\left(\mathrm{H}_{2} \mathrm{SO}_{4}\right)_{3} \cdot \mathrm{NH}_{3}$.

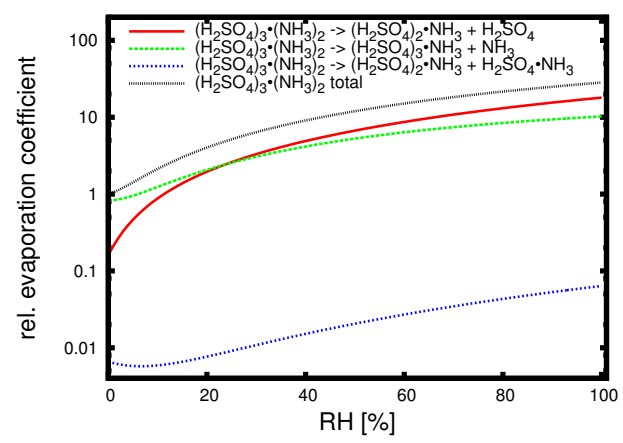

Figure S17: Relative evaporation coefficients for $\left(\mathrm{H}_{2} \mathrm{SO}_{4}\right)_{3} \cdot\left(\mathrm{NH}_{3}\right)_{2}$. 


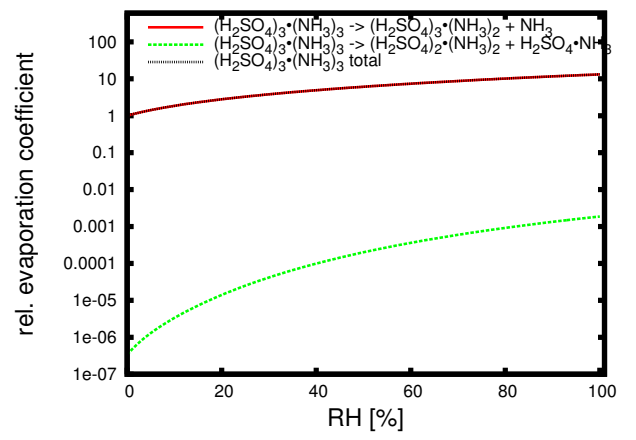

Figure S18: Relative evaporation coefficients for $\left(\mathrm{H}_{2} \mathrm{SO}_{4}\right)_{3} \cdot\left(\mathrm{NH}_{3}\right)_{3}$.

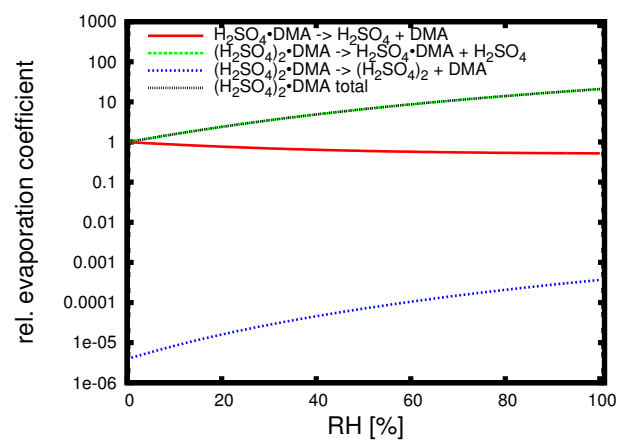

Figure S19: Relative evaporation coefficients for $\left(\mathrm{H}_{2} \mathrm{SO}_{4}\right)_{1,2} \cdot$ DMA.

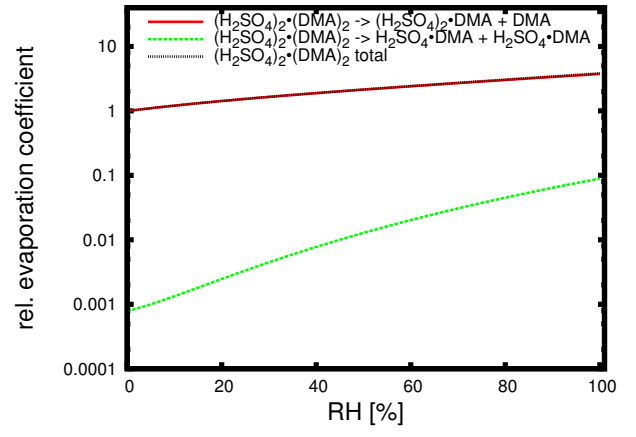

Figure S20: Relative evaporation coefficients for $\left(\mathrm{H}_{2} \mathrm{SO}_{4}\right)_{2} \cdot(\mathrm{DMA})_{2}$. 


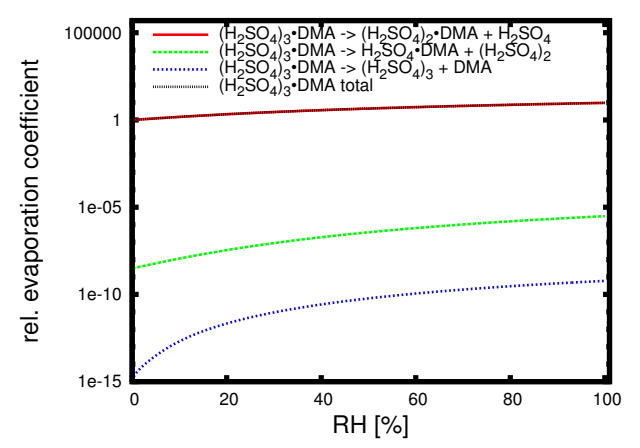

Figure S21: Relative evaporation coefficients for $\left(\mathrm{H}_{2} \mathrm{SO}_{4}\right)_{3} \cdot \mathrm{DMA}$.

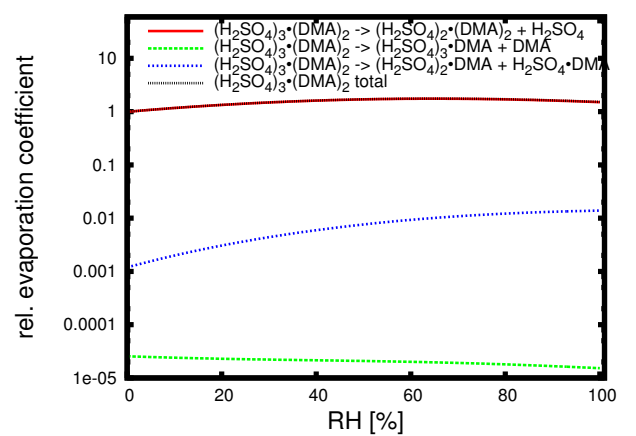

Figure S22: Relative evaporation coefficients for $\left(\mathrm{H}_{2} \mathrm{SO}_{4}\right)_{3} \cdot(\mathrm{DMA})_{2}$.

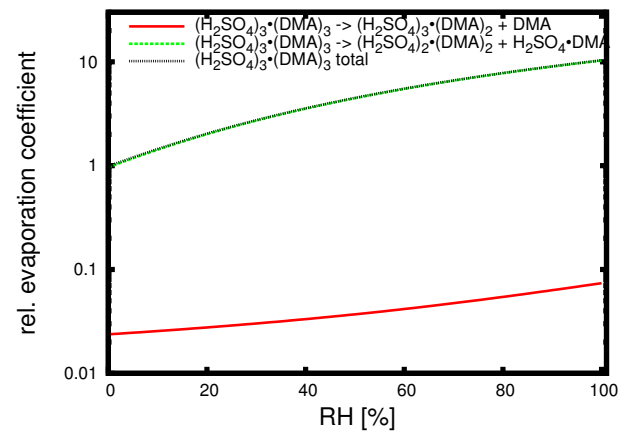

Figure S23: Relative evaporation coefficients for $\left(\mathrm{H}_{2} \mathrm{SO}_{4}\right)_{3} \cdot(\mathrm{DMA})_{3}$. 


\section{Particle Formation Rates}

\subsection{Base-Free System}

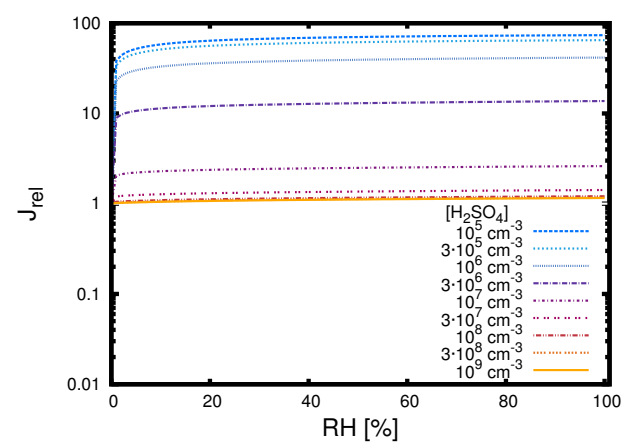

Figure S24: Relative particle formation rate at $T=193 \mathrm{~K}$.

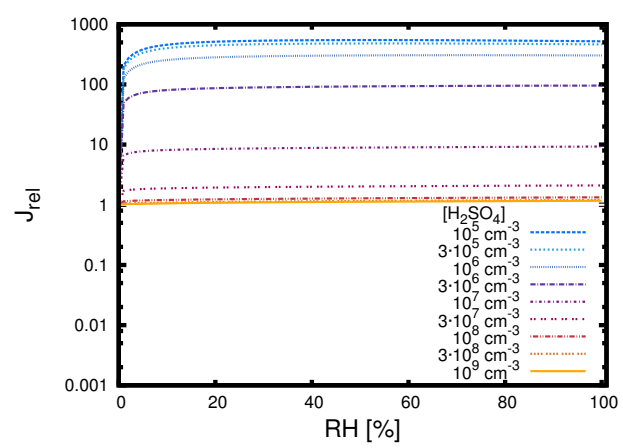

Figure S25: Relative particle formation rate at $T=198 \mathrm{~K}$.

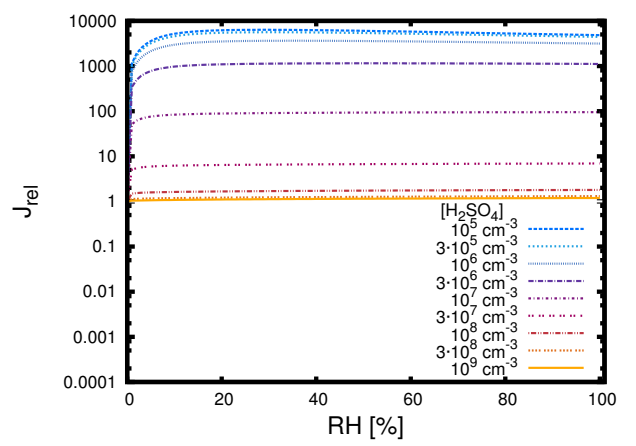

Figure S26: Relative particle formation rate at $T=203 \mathrm{~K}$. 


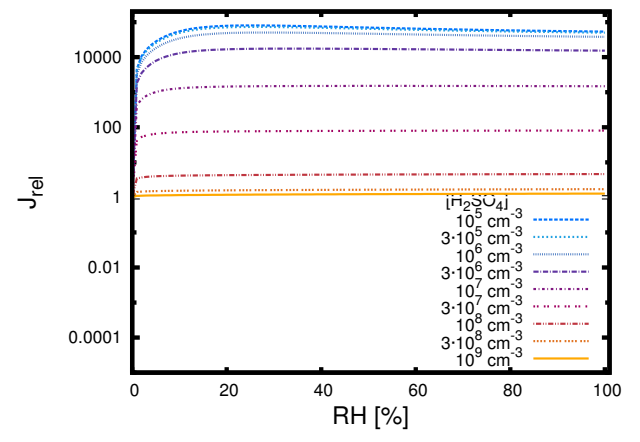

Figure S27: Relative particle formation rate at $T=208 \mathrm{~K}$.

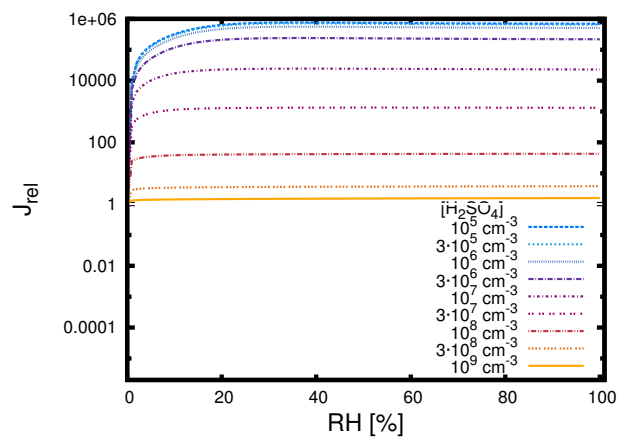

Figure S28: Relative particle formation rate at $T=213 \mathrm{~K}$.

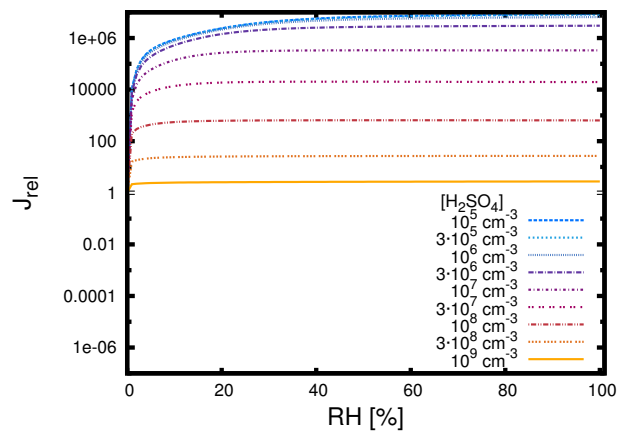

Figure S29: Relative particle formation rate at $T=218 \mathrm{~K}$. 


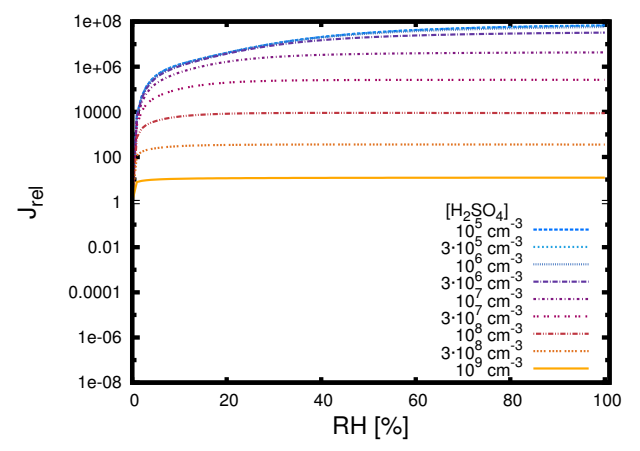

Figure S30: Relative particle formation rate at $T=223 \mathrm{~K}$.

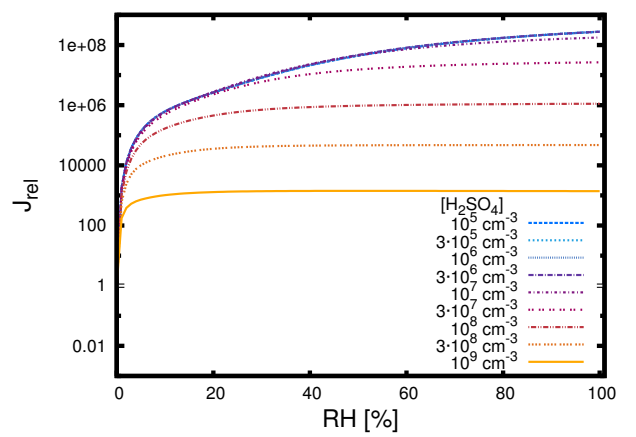

Figure S31: Relative particle formation rate at $T=233 \mathrm{~K}$.

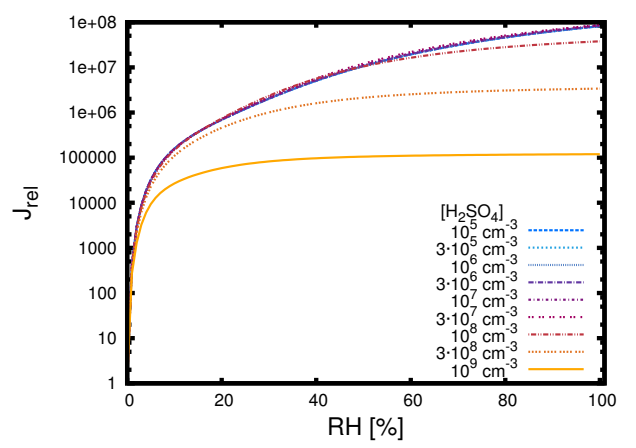

Figure S32: Relative particle formation rate at $T=243 \mathrm{~K}$. 


\section{$5.2 \mathrm{NH}_{3}$}

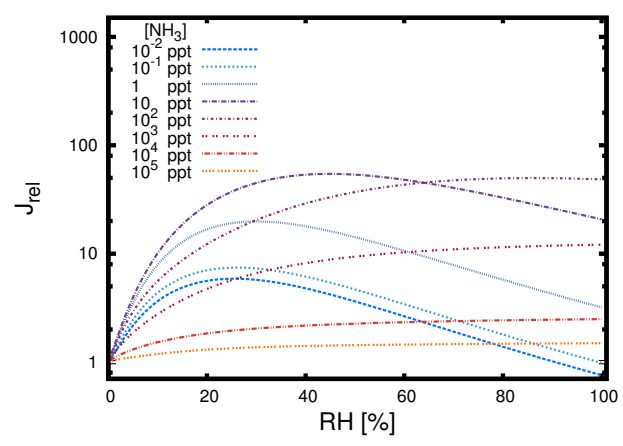

Figure S33: Relative particle formation rate at $T=263 \mathrm{~K}$, and $\left[\mathrm{H}_{2} \mathrm{SO}_{4}\right]=10^{5} \mathrm{~cm}^{-3}$.

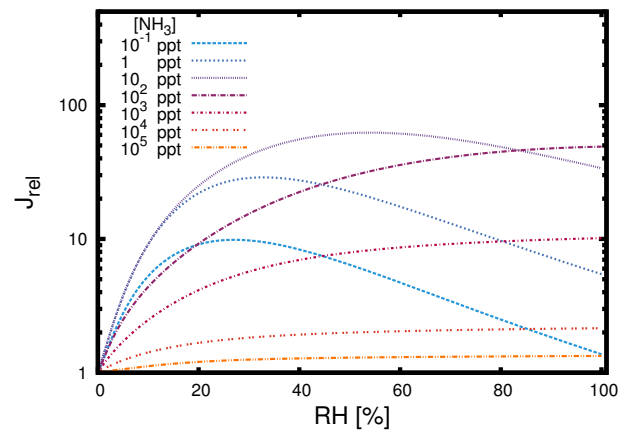

Figure S34: Relative particle formation rate at $T=263 \mathrm{~K}$, and $\left[\mathrm{H}_{2} \mathrm{SO}_{4}\right]=3 \cdot 10^{6} \mathrm{~cm}^{-3}$.

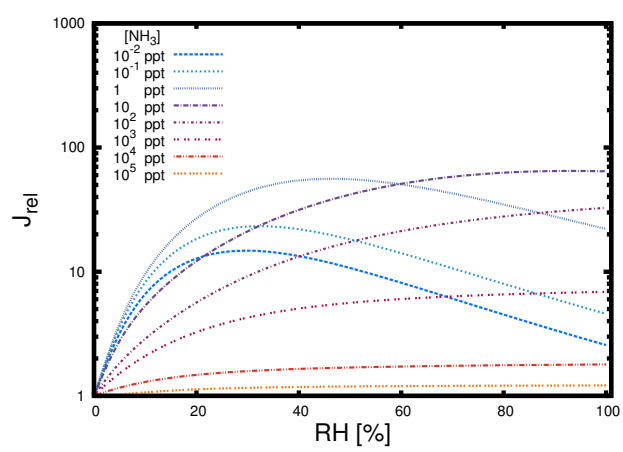

Figure S35: Relative particle formation rate at $T=263 \mathrm{~K}$, and $\left[\mathrm{H}_{2} \mathrm{SO}_{4}\right]=3 \cdot 10^{7} \mathrm{~cm}^{-3}$. 


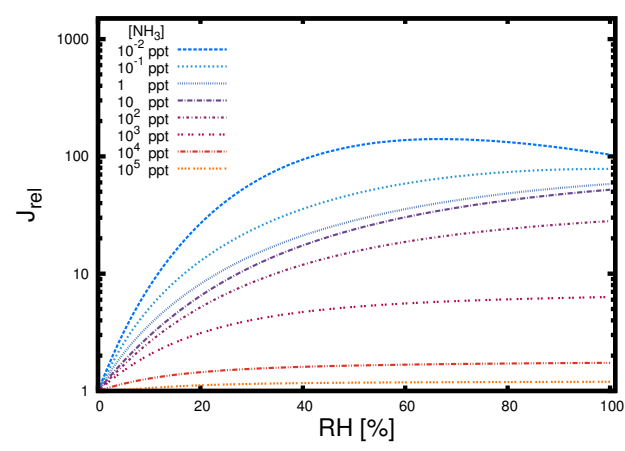

Figure S36: Relative particle formation rate at $T=263 \mathrm{~K}$, and $\left[\mathrm{H}_{2} \mathrm{SO}_{4}\right]=10^{9} \mathrm{~cm}^{-3}$.

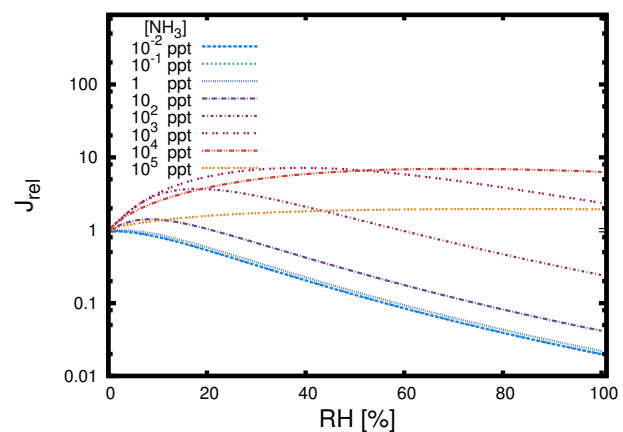

Figure S37: Relative particle formation rate at $T=278 \mathrm{~K}$, and $\left[\mathrm{H}_{2} \mathrm{SO}_{4}\right]=10^{5} \mathrm{~cm}^{-3}$.

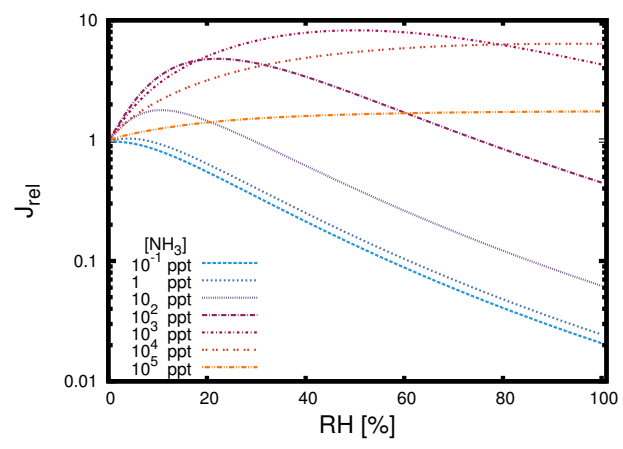

Figure S38: Relative particle formation rate at $T=278 \mathrm{~K}$, and $\left[\mathrm{H}_{2} \mathrm{SO}_{4}\right]=3 \cdot 10^{6} \mathrm{~cm}^{-3}$. 


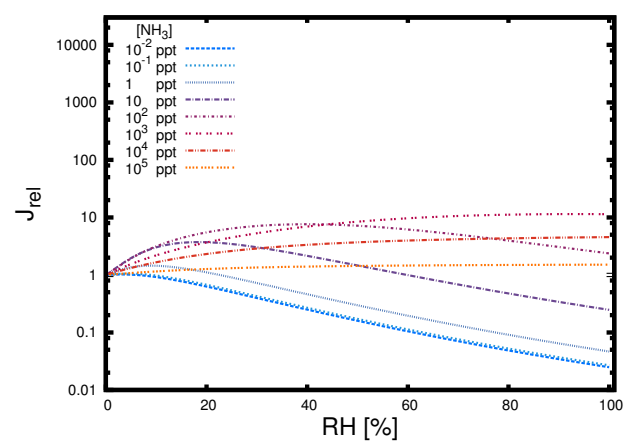

Figure S39: Relative particle formation rate at $T=278 \mathrm{~K}$, and $\left[\mathrm{H}_{2} \mathrm{SO}_{4}\right]=3 \cdot 10^{7} \mathrm{~cm}^{-3}$.

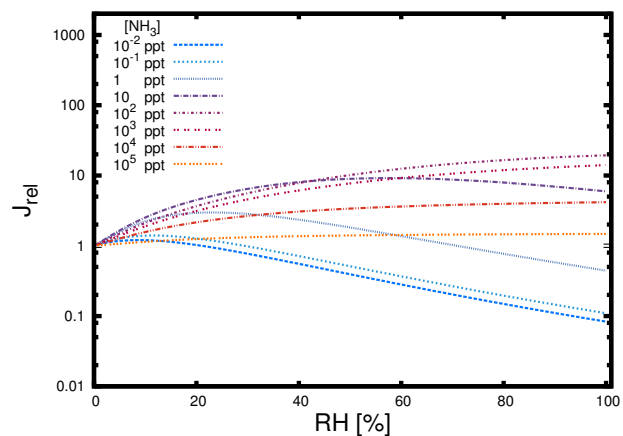

Figure S40: Relative particle formation rate at $T=278 \mathrm{~K}$, and $\left[\mathrm{H}_{2} \mathrm{SO}_{4}\right]=10^{9} \mathrm{~cm}^{-3}$.

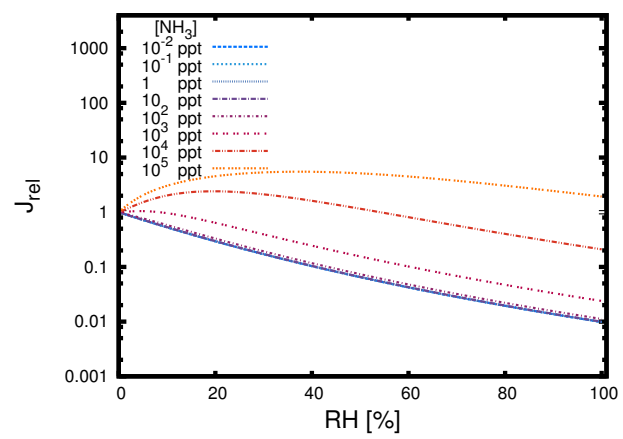

Figure S41: Relative particle formation rate at $T=293 \mathrm{~K}$, and $\left[\mathrm{H}_{2} \mathrm{SO}_{4}\right]=10^{5} \mathrm{~cm}^{-3}$. 


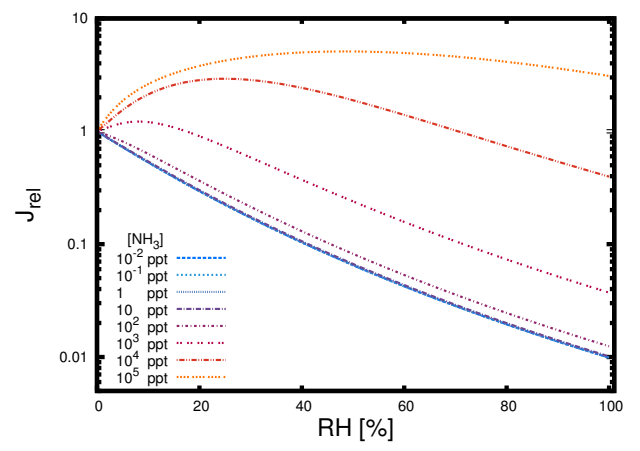

Figure S42: Relative particle formation rate at $T=293 \mathrm{~K}$, and $\left[\mathrm{H}_{2} \mathrm{SO}_{4}\right]=3 \cdot 10^{6} \mathrm{~cm}^{-3}$.

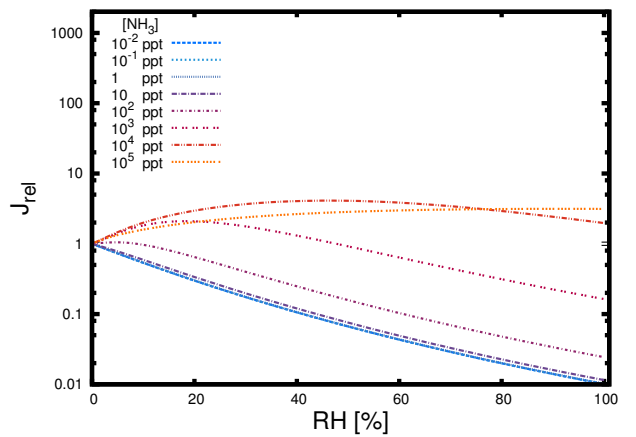

Figure S43: Relative particle formation rate at $T=293 \mathrm{~K}$, and $\left[\mathrm{H}_{2} \mathrm{SO}_{4}\right]=3 \cdot 10^{7} \mathrm{~cm}^{-3}$.

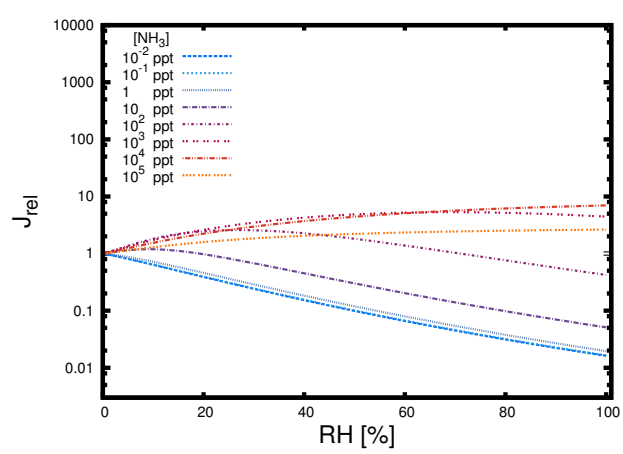

Figure S44: Relative particle formation rate at $T=293 \mathrm{~K}$, and $\left[\mathrm{H}_{2} \mathrm{SO}_{4}\right]=10^{9} \mathrm{~cm}^{-3}$. 


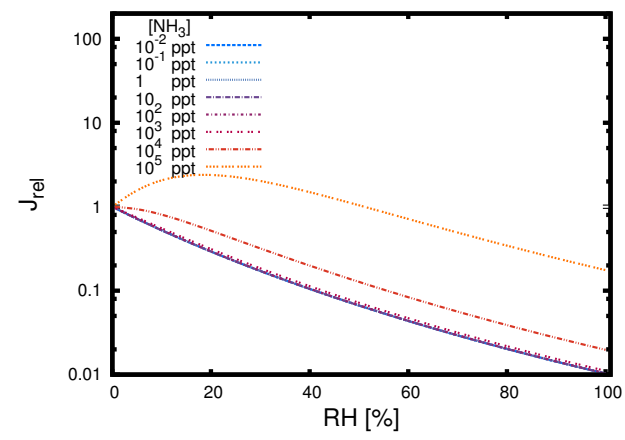

Figure S45: Relative particle formation rate at $T=303 \mathrm{~K}$, and $\left[\mathrm{H}_{2} \mathrm{SO}_{4}\right]=10^{5} \mathrm{~cm}^{-3}$.

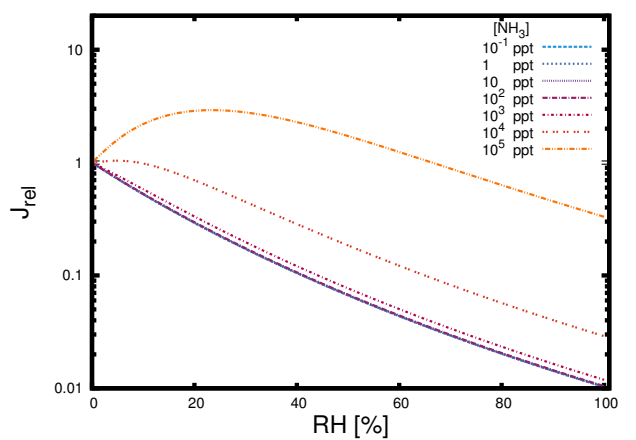

Figure S46: Relative particle formation rate at $T=303 \mathrm{~K}$, and $\left[\mathrm{H}_{2} \mathrm{SO}_{4}\right]=3 \cdot 10^{6} \mathrm{~cm}^{-3}$.

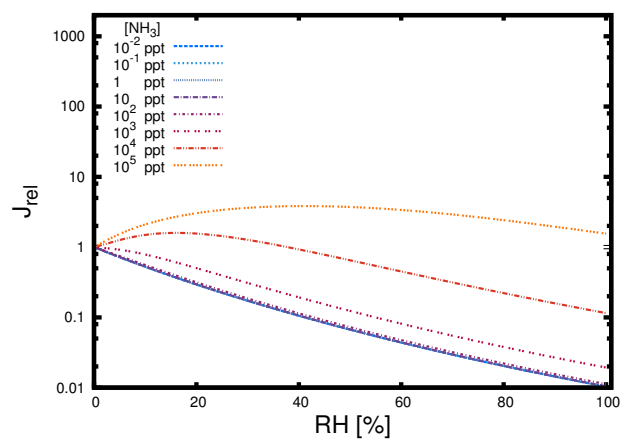

Figure S47: Relative particle formation rate at $T=303 \mathrm{~K}$, and $\left[\mathrm{H}_{2} \mathrm{SO}_{4}\right]=3 \cdot 10^{7} \mathrm{~cm}^{-3}$. 


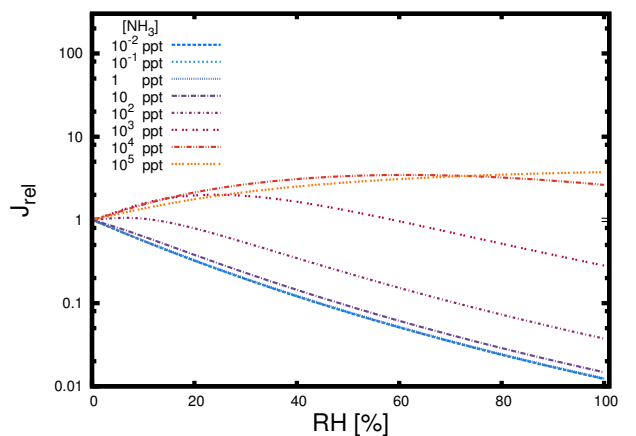

Figure S48: Relative particle formation rate at $T=303 \mathrm{~K}$, and $\left[\mathrm{H}_{2} \mathrm{SO}_{4}\right]=10^{9} \mathrm{~cm}^{-3}$. 


\subsection{DMA}

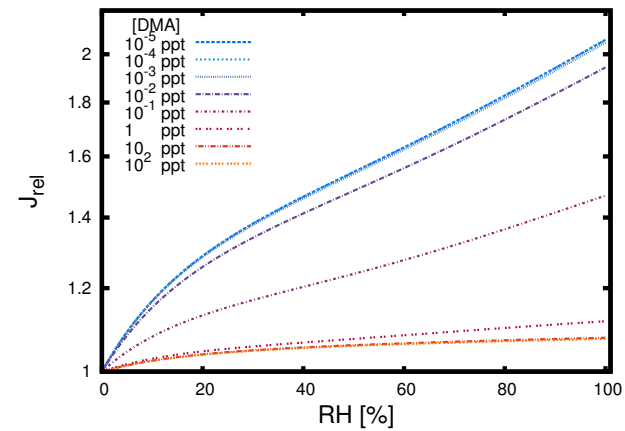

Figure S49: Relative particle formation rate at $T=263 \mathrm{~K}$, and $\left[\mathrm{H}_{2} \mathrm{SO}_{4}\right]=10^{5} \mathrm{~cm}^{-3}$.

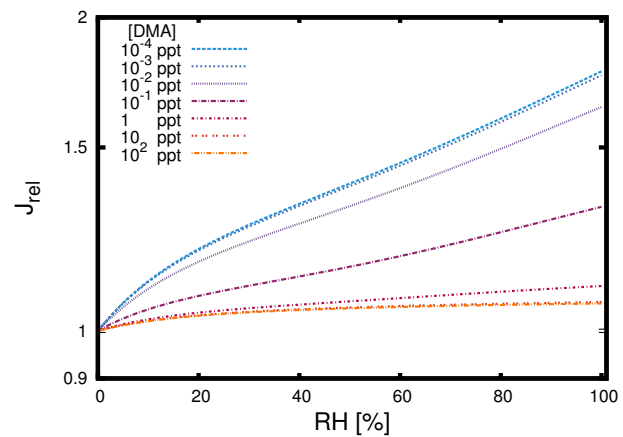

Figure S50: Relative particle formation rate at $T=263 \mathrm{~K}$, and $\left[\mathrm{H}_{2} \mathrm{SO}_{4}\right]=3 \cdot 10^{6} \mathrm{~cm}^{-3}$.

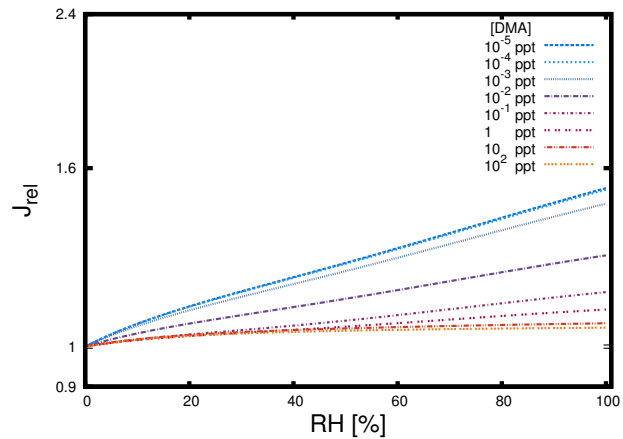

Figure S51: Relative particle formation rate at $T=263 \mathrm{~K}$, and $\left[\mathrm{H}_{2} \mathrm{SO}_{4}\right]=3 \cdot 10^{7} \mathrm{~cm}^{-3}$. 


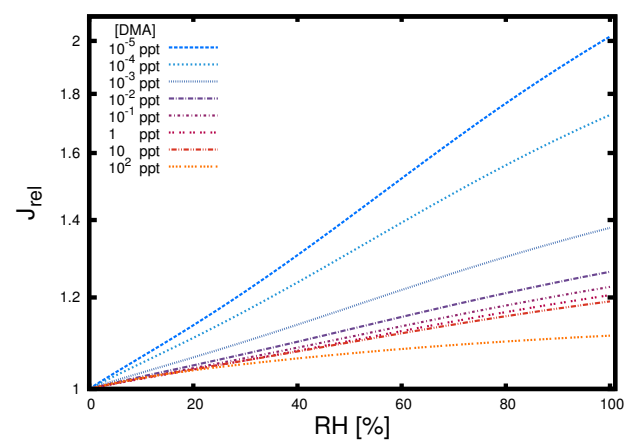

Figure S52: Relative particle formation rate at $T=263 \mathrm{~K}$, and $\left[\mathrm{H}_{2} \mathrm{SO}_{4}\right]=10^{9} \mathrm{~cm}^{-3}$.

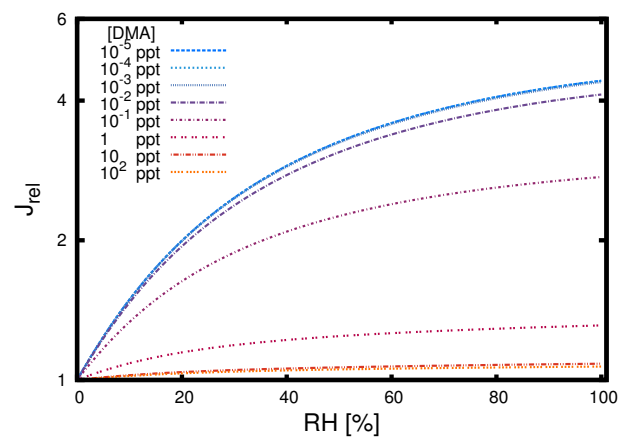

Figure S53: Relative particle formation rate at $T=278 \mathrm{~K}$, and $\left[\mathrm{H}_{2} \mathrm{SO}_{4}\right]=10^{5} \mathrm{~cm}^{-3}$.

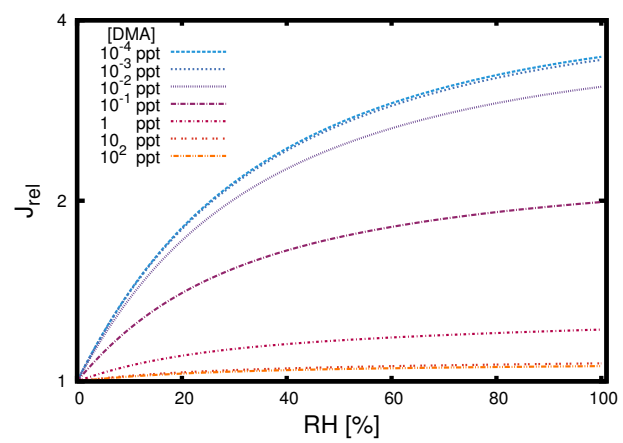

Figure S54: Relative particle formation rate at $T=278 \mathrm{~K}$, and $\left[\mathrm{H}_{2} \mathrm{SO}_{4}\right]=3 \cdot 10^{6} \mathrm{~cm}^{-3}$. 


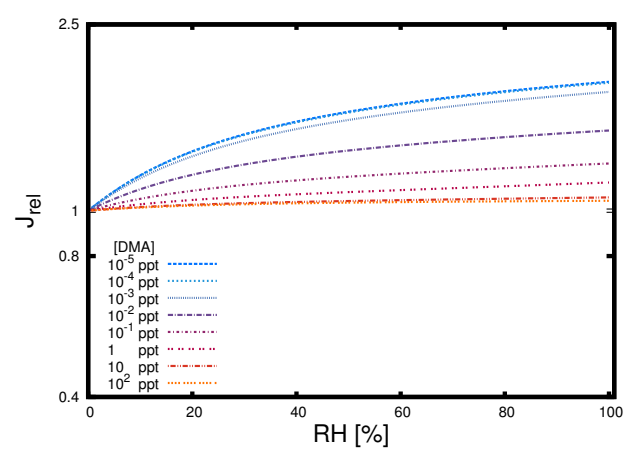

Figure S55: Relative particle formation rate at $T=278 \mathrm{~K}$, and $\left[\mathrm{H}_{2} \mathrm{SO}_{4}\right]=3 \cdot 10^{7} \mathrm{~cm}^{-3}$.

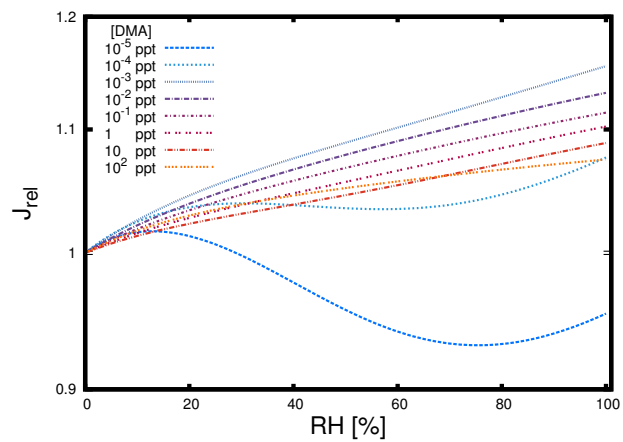

Figure S56: Relative particle formation rate at $T=278 \mathrm{~K}$, and $\left[\mathrm{H}_{2} \mathrm{SO}_{4}\right]=10^{9} \mathrm{~cm}^{-3}$.

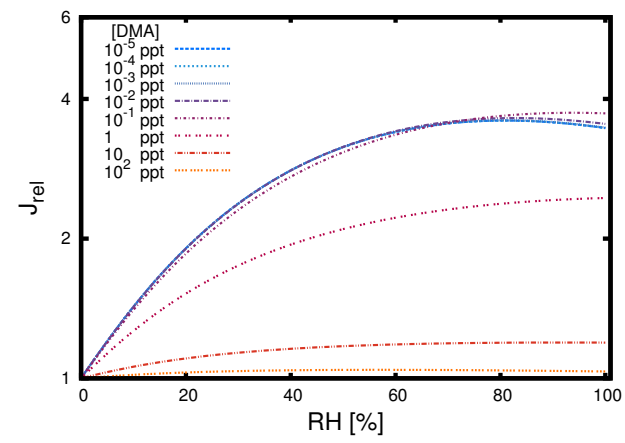

Figure S57: Relative particle formation rate at $T=293 \mathrm{~K}$, and $\left[\mathrm{H}_{2} \mathrm{SO}_{4}\right]=10^{5} \mathrm{~cm}^{-3}$. 


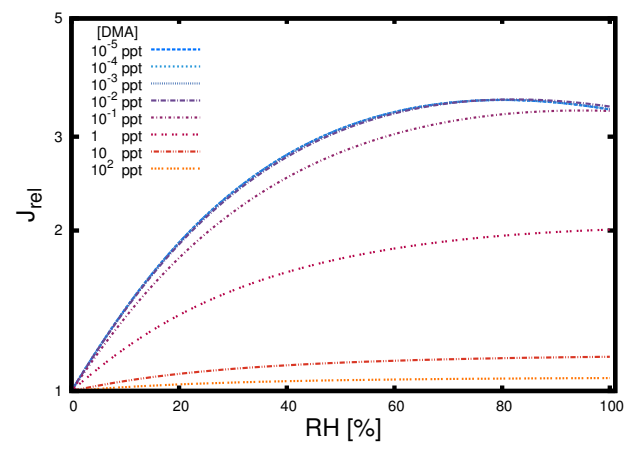

Figure S58: Relative particle formation rate at $T=293 \mathrm{~K}$, and $\left[\mathrm{H}_{2} \mathrm{SO}_{4}\right]=3 \cdot 10^{6} \mathrm{~cm}^{-3}$.

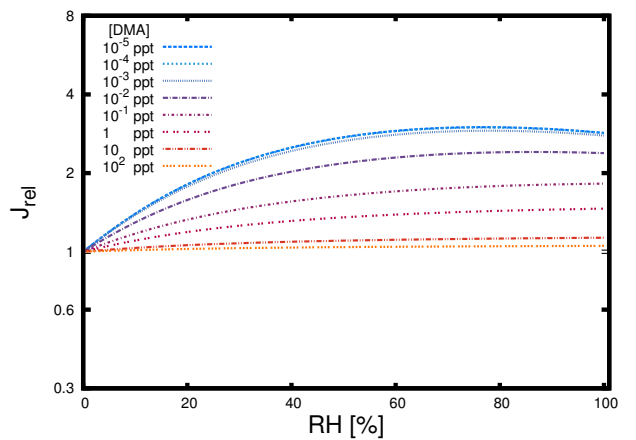

Figure S59: Relative particle formation rate at $T=293 \mathrm{~K}$, and $\left[\mathrm{H}_{2} \mathrm{SO}_{4}\right]=3 \cdot 10^{7} \mathrm{~cm}^{-3}$.

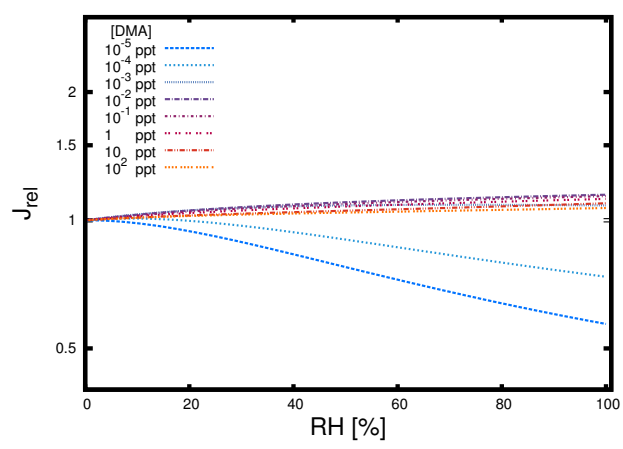

Figure S60: Relative particle formation rate at $T=293 \mathrm{~K}$, and $\left[\mathrm{H}_{2} \mathrm{SO}_{4}\right]=10^{9} \mathrm{~cm}^{-3}$. 


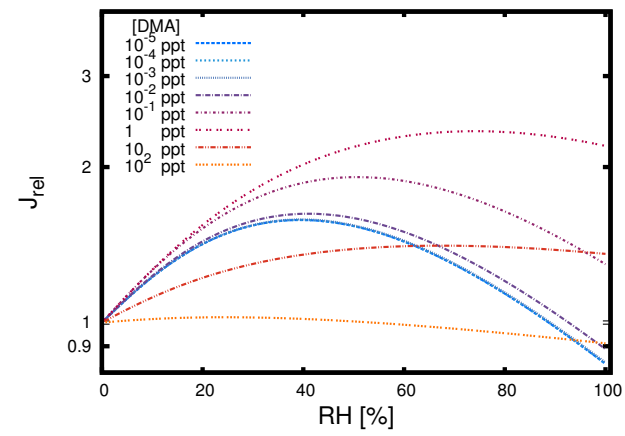

Figure S61: Relative particle formation rate at $T=303 \mathrm{~K}$, and $\left[\mathrm{H}_{2} \mathrm{SO}_{4}\right]=10^{5} \mathrm{~cm}^{-3}$.

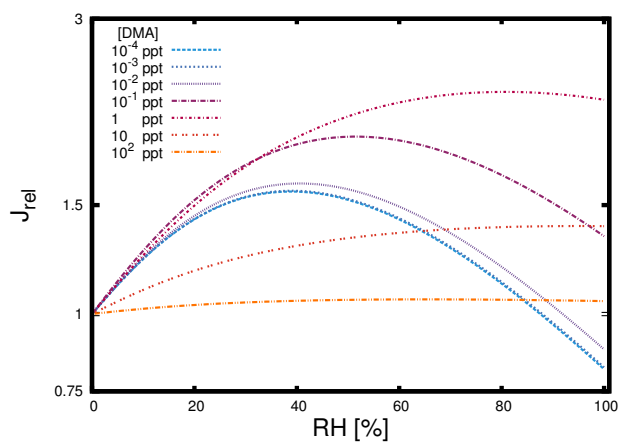

Figure S62: Relative particle formation rate at $T=303 \mathrm{~K}$, and $\left[\mathrm{H}_{2} \mathrm{SO}_{4}\right]=3 \cdot 10^{6} \mathrm{~cm}^{-3}$.

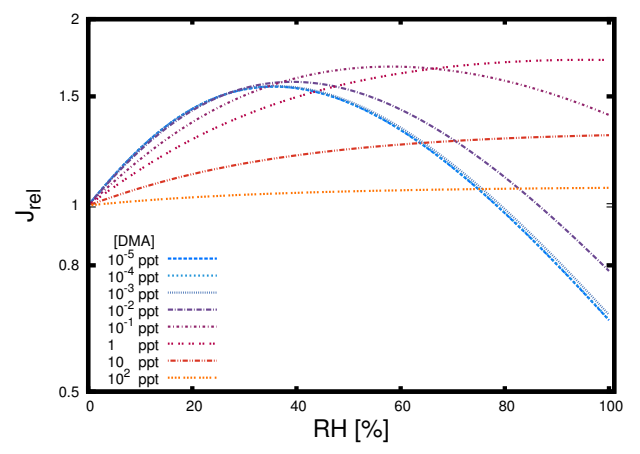

Figure S63: Relative particle formation rate at $T=303 \mathrm{~K}$, and $\left[\mathrm{H}_{2} \mathrm{SO}_{4}\right]=3 \cdot 10^{7} \mathrm{~cm}^{-3}$. 


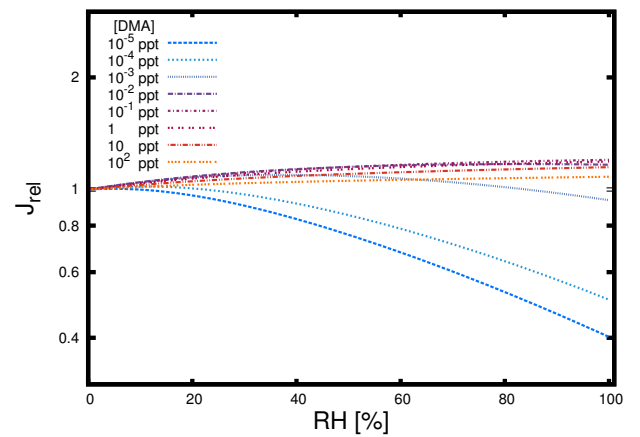

Figure S64: Relative particle formation rate at $T=303 \mathrm{~K}$, and $\left[\mathrm{H}_{2} \mathrm{SO}_{4}\right]=10^{9} \mathrm{~cm}^{-3}$. 


\section{Impact of Collision Rate Enhancement}

Results based on a 10-fold increase of collision coefficients.

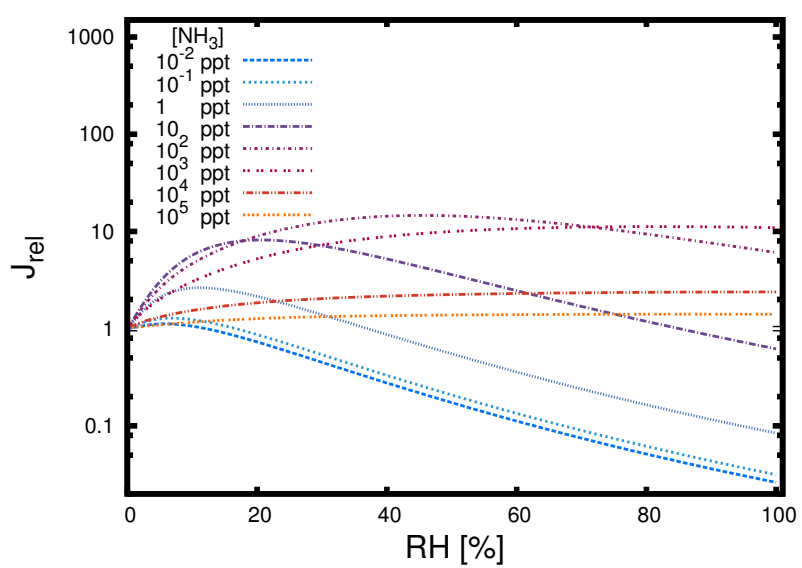

(a) $\left[\mathrm{H}_{2} \mathrm{SO}_{4}\right]=10^{5} \mathrm{~cm}^{-3}$, base $=\mathrm{NH}_{3}$.

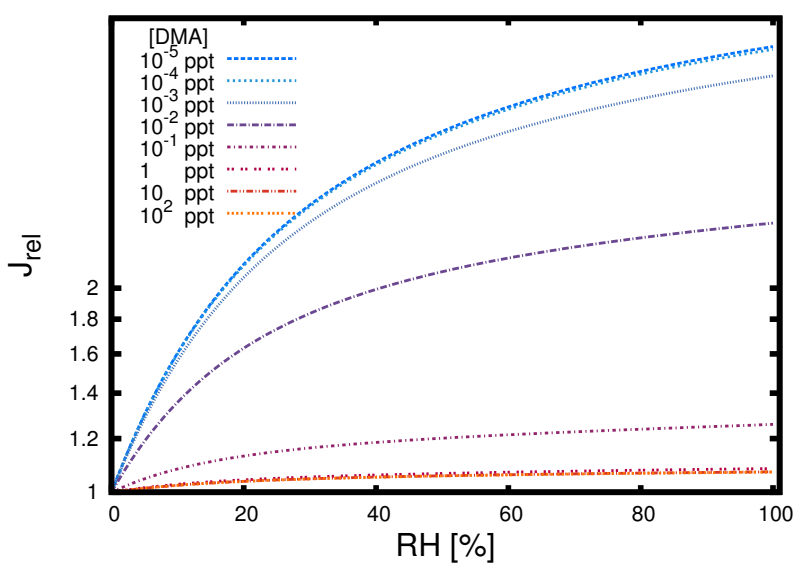

(c) $\left[\mathrm{H}_{2} \mathrm{SO}_{4}\right]=10^{5} \mathrm{~cm}^{-3}$, base $=$ DMA.

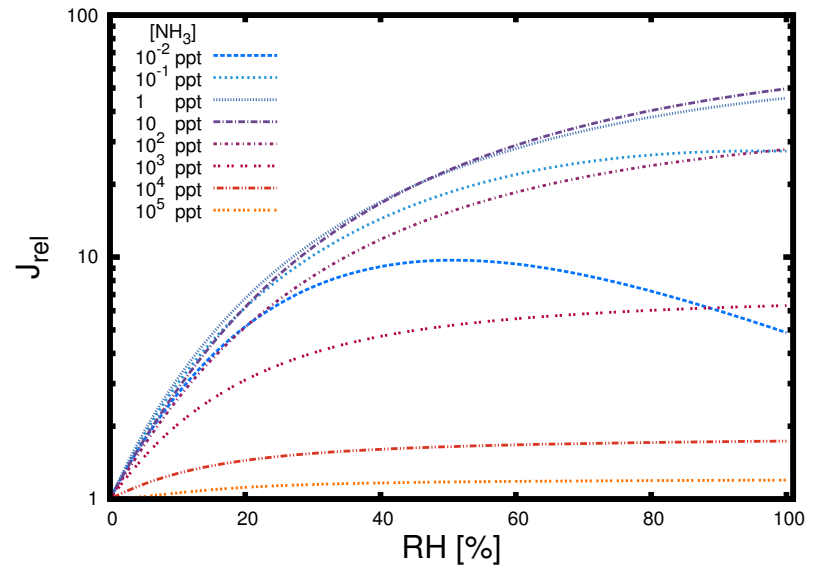

(b) $\left[\mathrm{H}_{2} \mathrm{SO}_{4}\right]=10^{9} \mathrm{~cm}^{-3}$, base $=\mathrm{NH}_{3}$.

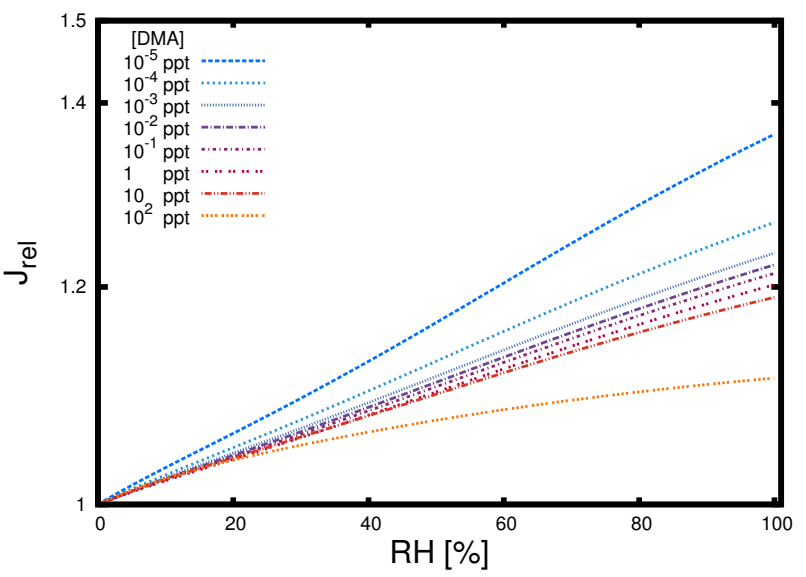

(d) $\left[\mathrm{H}_{2} \mathrm{SO}_{4}\right]=10^{9} \mathrm{~cm}^{-3}$, base $=$ DMA.

Figure S65: Modelled relative particle formation rate as function of relative humidity at $263 \mathrm{~K}$. 


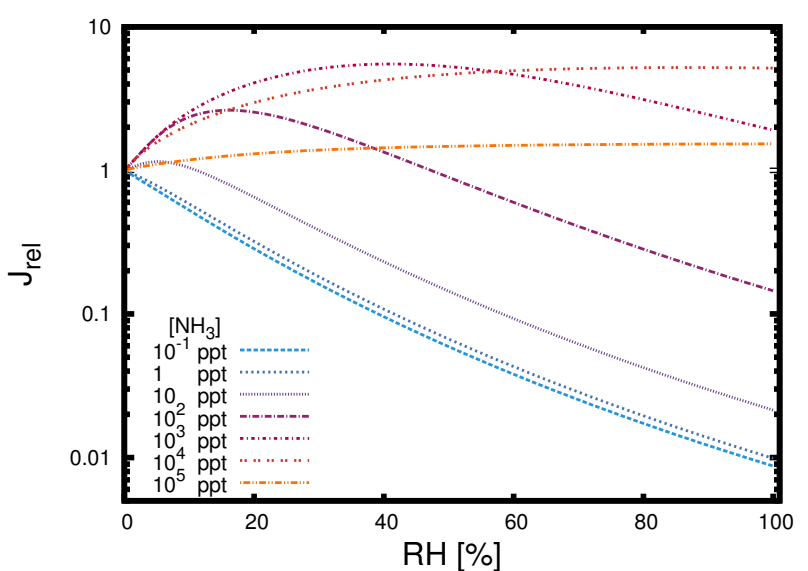

(a) $T=278 \mathrm{~K},\left[\mathrm{H}_{2} \mathrm{SO}_{4}\right]=3 \cdot 10^{6} \mathrm{~cm}^{-3}$, base $=\mathrm{NH}_{3}$.

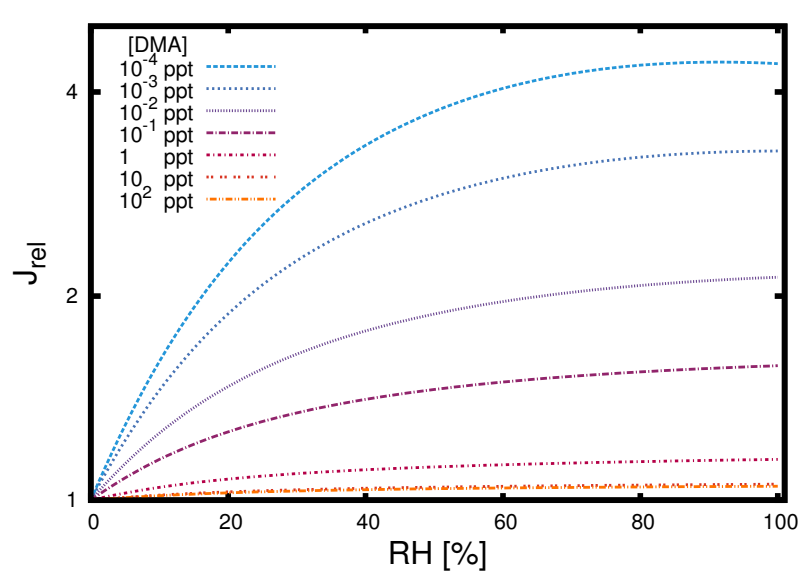

(c) $T=278 \mathrm{~K},\left[\mathrm{H}_{2} \mathrm{SO}_{4}\right]=3 \cdot 10^{6} \mathrm{~cm}^{-3}$, base $=$ DMA.

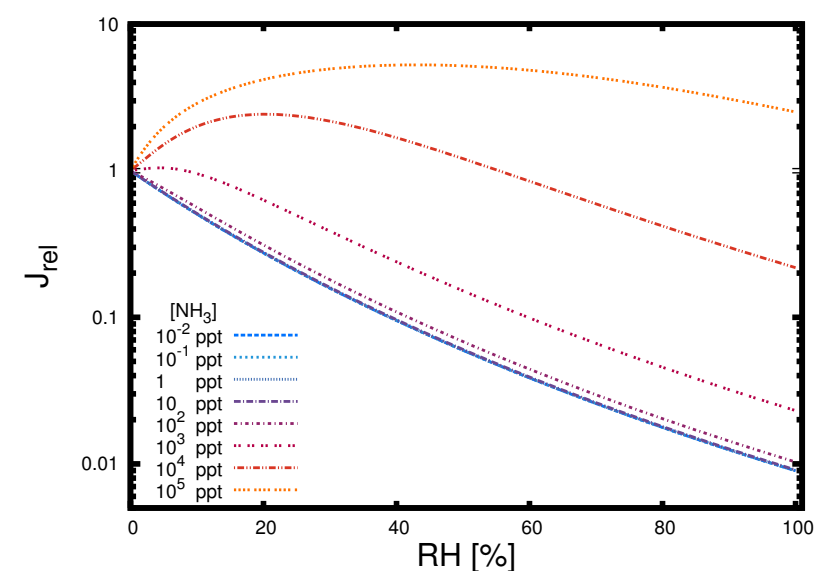

(b) $T=293 \mathrm{~K},\left[\mathrm{H}_{2} \mathrm{SO}_{4}\right]=3 \cdot 10^{6} \mathrm{~cm}^{-3}$, base $=\mathrm{NH}_{3}$.

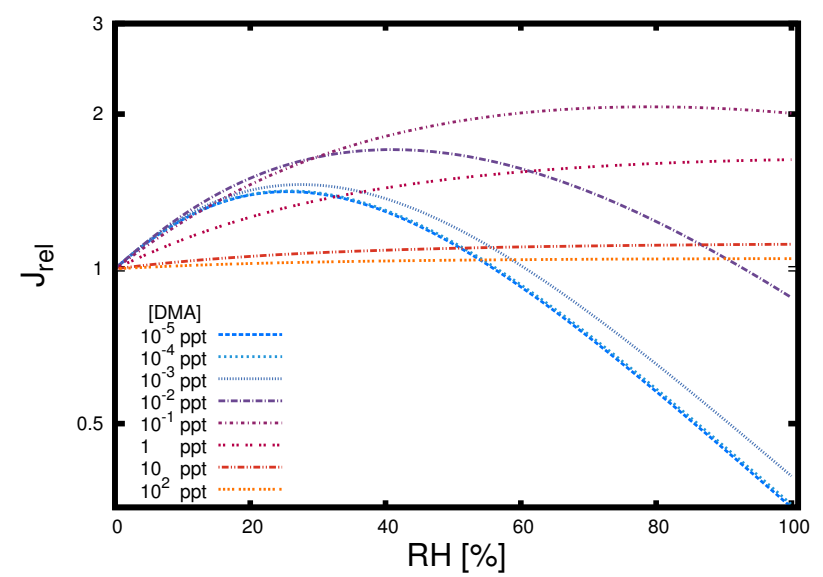

(d) $T=293 \mathrm{~K},\left[\mathrm{H}_{2} \mathrm{SO}_{4}\right]=3 \cdot 10^{6} \mathrm{~cm}^{-3}$, base = DMA.

Figure S66: Modelled relative particle formation rate as function of relative humidity at intermediate temperatures and sulfuric acid concentrations. 


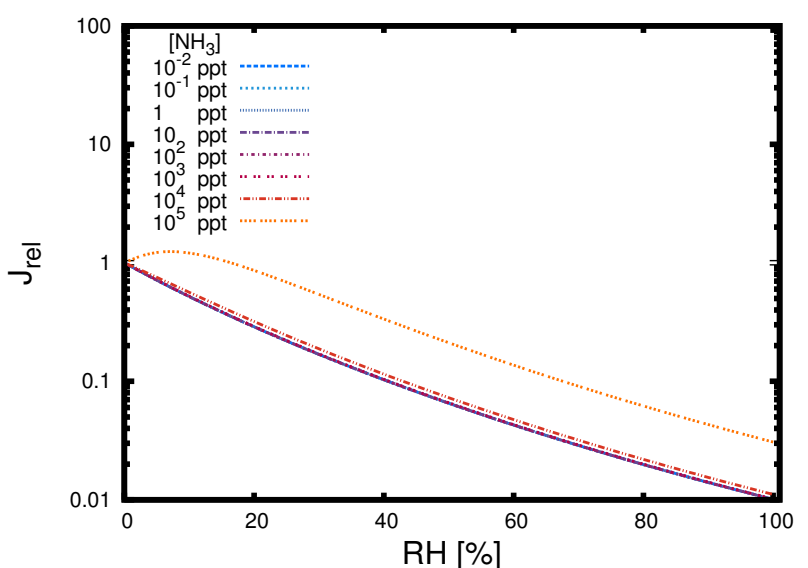

(a) $\left[\mathrm{H}_{2} \mathrm{SO}_{4}\right]=10^{5} \mathrm{~cm}^{-3}$, base $=\mathrm{NH}_{3}$.

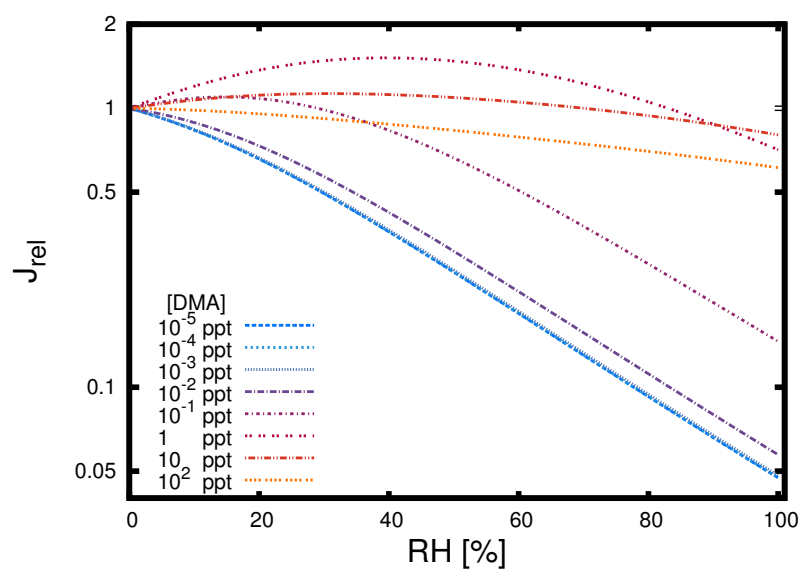

(c) $\left[\mathrm{H}_{2} \mathrm{SO}_{4}\right]=10^{5} \mathrm{~cm}^{-3}$, base $=$ DMA.

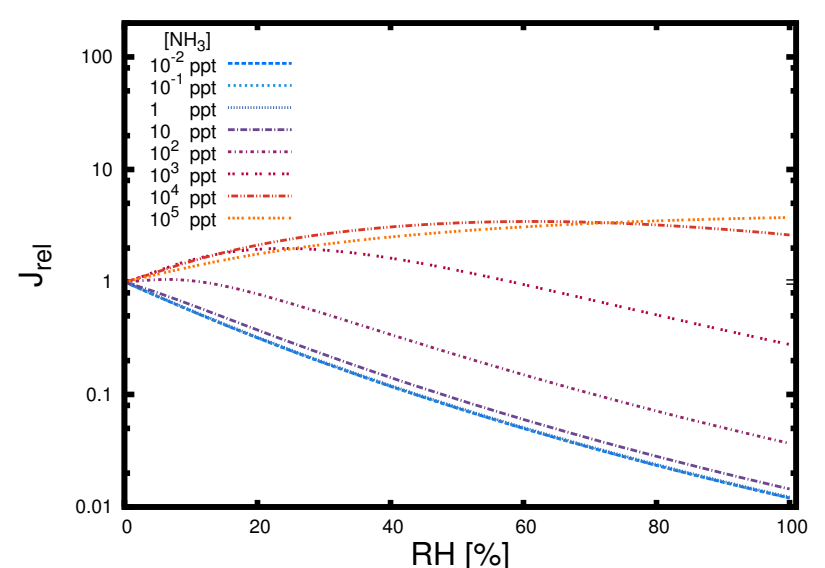

(b) $\left[\mathrm{H}_{2} \mathrm{SO}_{4}\right]=10^{9} \mathrm{~cm}^{-3}$, base $=\mathrm{NH}_{3}$.

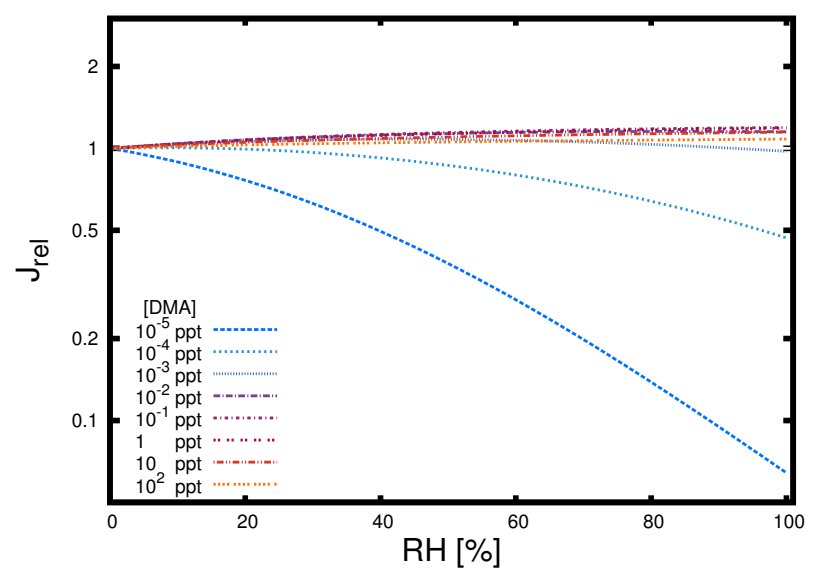

(d) $\left[\mathrm{H}_{2} \mathrm{SO}_{4}\right]=10^{9} \mathrm{~cm}^{-3}$, base $=$ DMA.

Figure S67: Modelled relative particle formation rate as function of relative humidity at $303 \mathrm{~K}$. 


\section{Cartesian Coordinates of Optimized Structures}

All coordinates in $\AA$.

\section{$7.1\left(\mathrm{H}_{2} \mathrm{SO}_{4}\right)_{3} \cdot\left(\mathrm{NH}_{3}\right)_{3}$}

$\begin{array}{rrrr}\mathrm{S} & 2.797158000 & -1.259676000 & 0.043302000 \\ \mathrm{O} & 1.748610000 & -2.278489000 & -0.014088000 \\ \mathrm{O} & 4.202316000 & -2.059527000 & 0.221424000 \\ \mathrm{O} & 3.007899000 & -0.529218000 & -1.207582000 \\ \mathrm{H} & 4.080880000 & -2.716772000 & 0.921156000 \\ \mathrm{~S} & -3.007693000 & -1.008475000 & -0.030646000 \\ \mathrm{O} & -3.204995000 & 0.421544000 & 0.145509000 \\ \mathrm{O} & -4.438939000 & -1.619705000 & 0.431692000 \\ \mathrm{O} & -2.814099000 & -1.457240000 & -1.418224000 \\ \mathrm{H} & -4.445859000 & -2.555074000 & 0.185768000 \\ \mathrm{~S} & -0.327449000 & 2.284260000 & -0.018648000 \\ \mathrm{O} & 0.177004000 & 0.944505000 & -0.410533000 \\ \mathrm{O} & -0.559873000 & 2.340288000 & 1.428903000 \\ \mathrm{O} & -1.709733000 & 2.504106000 & -0.761503000 \\ \mathrm{O} & 0.546333000 & 3.344446000 & -0.529886000 \\ \mathrm{H} & 2.067957000 & 2.646197000 & -0.533388000 \\ \mathrm{O} & 2.669919000 & -0.376371000 & 1.218447000 \\ \mathrm{~N} & -0.070616000 & -0.231713000 & 2.253968000 \\ \mathrm{~N} & 2.956723000 & 2.087148000 & -0.396379000 \\ \mathrm{~N} & -0.125633000 & -1.300552000 & -1.892332000 \\ \mathrm{H} & 3.784620000 & 2.645015000 & -0.589643000 \\ \mathrm{H} & 2.982219000 & 1.728799000 & 0.563791000 \\ \mathrm{H} & 2.945073000 & 1.215047000 & -0.970023000 \\ \mathrm{H} & -2.337856000 & 1.795672000 & -0.463498000 \\ \mathrm{H} & 0.467529000 & -1.882465000 & -1.274253000 \\ \mathrm{H} & 0.189170000 & -1.381204000 & -2.855151000 \\ \mathrm{H} & -1.141278000 & -1.539131000 & -1.795711000 \\ \mathrm{H} & -0.015001000 & -0.321717000 & -1.548227000 \\ \mathrm{H} & 0.878588000 & -0.414745000 & 1.887370000 \\ \mathrm{H} & -0.782532000 & -0.793653000 & 1.728169000 \\ \mathrm{H} & -0.289082000 & 0.779502000 & 2.083149000 \\ \mathrm{H} & -0.117295000 & -0.450468000 & 3.245551000 \\ \mathrm{O} & -1.997277000 & -1.596455000 & 0.865096000\end{array}$

$7.2\left(\mathrm{H}_{2} \mathrm{SO}_{4}\right)_{3} \cdot\left(\mathrm{NH}_{3}\right)_{3} \cdot \mathrm{H}_{2} \mathrm{O}$

S $\quad-0.039506000$

0.161852000

$-0.020308000$ 


$\begin{array}{lrrr}\mathrm{O} & 0.098171000 & 0.094901000 & 1.442647000 \\ \mathrm{O} & 1.473014000 & 0.095560000 & -0.609331000 \\ \mathrm{O} & -0.681613000 & -0.980837000 & -0.645015000 \\ \mathrm{H} & 2.007524000 & 0.729069000 & -0.111128000 \\ \mathrm{~S} & -3.961291000 & 4.264534000 & 1.131672000 \\ \mathrm{O} & -4.643313000 & 3.735809000 & -0.072709000 \\ \mathrm{O} & -4.143036000 & 5.879760000 & 1.076130000 \\ \mathrm{O} & -4.635004000 & 3.905597000 & 2.373962000 \\ \mathrm{H} & -3.531837000 & 6.221999000 & 0.408828000 \\ \mathrm{~S} & -4.884998000 & -0.401972000 & 0.720443000 \\ \mathrm{O} & -4.567969000 & -0.581810000 & -0.706899000 \\ \mathrm{O} & -4.463893000 & -1.714858000 & 1.472479000 \\ \mathrm{O} & -6.317533000 & -0.230671000 & 1.003160000 \\ \mathrm{O} & -4.112270000 & 0.727404000 & 1.289578000 \\ \mathrm{H} & -6.049660000 & 2.763907000 & 2.088427000 \\ \mathrm{O} & -0.593697000 & 1.460033000 & -0.444624000 \\ \mathrm{~N} & -3.129370000 & 1.681900000 & -1.438948000 \\ \mathrm{~N} & -6.715528000 & 2.383650000 & 1.390670000 \\ \mathrm{~N} & -1.889244000 & 1.658935000 & 2.523573000 \\ \mathrm{O} & -2.566753000 & -2.830859000 & 0.125819000 \\ \mathrm{H} & -1.933839000 & 2.585660000 & 2.059663000 \\ \mathrm{H} & -2.744147000 & 1.143954000 & 2.217154000 \\ \mathrm{H} & -1.049869000 & 1.122714000 & 2.199101000 \\ \mathrm{H} & -6.397428000 & 2.825127000 & 0.518282000 \\ \mathrm{H} & -6.615087000 & 1.326380000 & 1.302010000 \\ \mathrm{H} & -1.791328000 & -2.247971000 & -0.003399000 \\ \mathrm{H} & -2.967769000 & -2.875093000 & -0.749777000 \\ \mathrm{H} & -3.686078000 & -2.161576000 & 0.988401000 \\ \mathrm{H} & -2.145240000 & 1.600418000 & -1.082404000 \\ \mathrm{H} & -3.614931000 & 2.458770000 & -0.954034000 \\ \mathrm{H} & -3.641655000 & 0.791515000 & -1.235326000 \\ \mathrm{O} & -2.519992000 & 4.017718000 & 1.104755000 \\ \mathrm{H} & -1.886736000 & 1.773418000 & 3.533495000 \\ \mathrm{H} & -3.114221000 & 1.853629000 & -2.441090000 \\ \mathrm{H} & -7.670808000 & 2.654346000 & 1.608459000\end{array}$

\section{$7.3\left(\mathrm{H}_{2} \mathrm{SO}_{4}\right)_{3} \cdot\left(\mathrm{NH}_{3}\right)_{3} \cdot\left(\mathrm{H}_{2} \mathrm{O}\right)_{2}$}

$\begin{array}{lrrr}\mathrm{S} & -0.090565000 & 0.115884000 & 0.125199000 \\ \mathrm{O} & -0.309552000 & -0.086529000 & 1.580514000 \\ \mathrm{O} & 1.519152000 & 0.134137000 & -0.089078000 \\ \mathrm{O} & -0.537828000 & -1.001235000 & -0.678652000 \\ \mathrm{H} & 1.818074000 & 1.048006000 & 0.012215000\end{array}$




$\begin{array}{lrrr}\mathrm{S} & -3.950705000 & 4.414143000 & 0.689275000 \\ \mathrm{O} & -4.652947000 & 3.691360000 & -0.385645000 \\ \mathrm{O} & -4.115545000 & 5.959285000 & 0.207241000 \\ \mathrm{O} & -4.570913000 & 4.311643000 & 2.002890000 \\ \mathrm{H} & -3.905197000 & 6.522448000 & 0.964889000 \\ \mathrm{~S} & -4.677283000 & -0.286405000 & 1.378079000 \\ \mathrm{O} & -4.295510000 & -0.636248000 & -0.017828000 \\ \mathrm{O} & -4.391477000 & -1.376632000 & 2.310273000 \\ \mathrm{O} & -6.229886000 & -0.073745000 & 1.413917000 \\ \mathrm{O} & -4.074434000 & 1.004804000 & 1.756116000 \\ \mathrm{H} & -6.184889000 & 2.926882000 & 1.884993000 \\ \mathrm{O} & -0.554475000 & 1.444173000 & -0.294431000 \\ \mathrm{~N} & -3.185223000 & 1.548457000 & -1.331424000 \\ \mathrm{O} & -6.720561000 & 2.464032000 & 1.220661000 \\ \mathrm{~N} & -1.819697000 & 2.197861000 & 2.515079000 \\ \mathrm{O} & -2.647898000 & -2.889542000 & -0.576767000 \\ \mathrm{H} & -1.910958000 & 2.990677000 & 1.835980000 \\ \mathrm{H} & -2.722302000 & 1.671653000 & 2.443896000 \\ \mathrm{H} & -1.070873000 & 1.543822000 & 2.236345000 \\ \mathrm{H} & -6.347985000 & 2.828652000 & 0.401833000 \\ \mathrm{H} & -6.447366000 & 0.919906000 & 1.330846000 \\ \mathrm{H} & -1.943309000 & -2.359566000 & -0.979882000 \\ \mathrm{H} & -3.403571000 & -2.274876000 & -0.522485000 \\ \mathrm{H} & -2.099280000 & -2.790472000 & 0.990660000 \\ \mathrm{H} & -2.198161000 & 1.605570000 & -1.015623000 \\ \mathrm{H} & -3.703495000 & 2.406662000 & -1.030655000 \\ \mathrm{H} & -3.630462000 & 0.711696000 & -0.884043000 \\ \mathrm{H} & -3.214929000 & 1.458654000 & -2.344033000 \\ \mathrm{O} & -2.506000000 & 4.134433000 & 0.694667000 \\ \mathrm{H} & -2.821721000 & -2.061642000 & 2.305639000 \\ \mathrm{~N} & -1.905072000 & -2.448563000 & 1.978043000 \\ \mathrm{H} & -1.212045000 & -1.679519000 & 1.927253000 \\ \mathrm{H} & -1.676532000 & 2.551244000 & 3.456985000 \\ \mathrm{H} & -1.578989000 & -3.198798000 & 2.580610000\end{array}$

\section{$7.4\left(\mathrm{H}_{2} \mathrm{SO}_{4}\right)_{3} \cdot\left(\mathrm{NH}_{3}\right)_{3} \cdot\left(\mathrm{H}_{2} \mathrm{O}\right)_{3}$}

$\begin{array}{rrrr}\mathrm{S} & -0.214098000 & 0.585310000 & -0.178787000 \\ \mathrm{O} & 0.001555000 & 0.557093000 & 1.297563000 \\ \mathrm{O} & 1.209360000 & 0.606185000 & -0.857608000 \\ \mathrm{O} & -0.860613000 & -0.613405000 & -0.686708000 \\ \mathrm{H} & 1.687762000 & 1.466583000 & -0.629957000 \\ \mathrm{~S} & -4.500682000 & 4.265447000 & 1.040923000\end{array}$




$\begin{array}{lrrr}\mathrm{O} & -5.177123000 & 3.636491000 & -0.118019000 \\ \mathrm{O} & -4.876459000 & 5.846429000 & 0.973526000 \\ \mathrm{O} & -5.058578000 & 3.854748000 & 2.323359000 \\ \mathrm{H} & -4.371905000 & 6.239154000 & 0.247432000 \\ \mathrm{~S} & -4.969624000 & -0.496035000 & 0.794580000 \\ \mathrm{O} & -4.672613000 & -0.671559000 & -0.635749000 \\ \mathrm{O} & -4.412013000 & -1.746689000 & 1.562496000 \\ \mathrm{O} & -6.404893000 & -0.455744000 & 1.112683000 \\ \mathrm{O} & -4.295725000 & 0.714160000 & 1.324274000 \\ \mathrm{H} & -6.379458000 & 2.564457000 & 2.132070000 \\ \mathrm{O} & -0.906063000 & 1.835737000 & -0.553607000 \\ \mathrm{~N} & -3.480174000 & 1.694938000 & -1.468635000 \\ \mathrm{~N} & -7.026868000 & 2.115717000 & 1.459860000 \\ \mathrm{~N} & -2.137390000 & 1.938017000 & 2.397549000 \\ \mathrm{O} & -2.338362000 & -2.670287000 & 0.337749000 \\ \mathrm{H} & -2.286406000 & 2.839053000 & 1.905695000 \\ \mathrm{H} & -2.948590000 & 1.329861000 & 2.143545000 \\ \mathrm{H} & -1.262120000 & 1.476080000 & 2.059521000 \\ \mathrm{H} & -6.770945000 & 2.568408000 & 0.572047000 \\ \mathrm{H} & -6.835107000 & 1.069123000 & 1.386378000 \\ \mathrm{H} & -1.703968000 & -1.962684000 & 0.097423000 \\ \mathrm{H} & -2.674008000 & -2.979879000 & -0.510583000 \\ \mathrm{H} & -3.574852000 & -2.103243000 & 1.103176000 \\ \mathrm{H} & -2.483751000 & 1.749178000 & -1.140263000 \\ \mathrm{H} & -4.041247000 & 2.431602000 & -1.004754000 \\ \mathrm{H} & -3.880218000 & 0.760654000 & -1.213818000 \\ \mathrm{O} & -3.043592000 & 4.187198000 & 0.938929000 \\ \mathrm{O} & 2.434758000 & 2.776473000 & -0.065281000 \\ \mathrm{H} & 1.850089000 & 3.539946000 & -0.112430000 \\ \mathrm{H} & 2.503131000 & 2.545977000 & 0.890261000 \\ \mathrm{H} & -3.510652000 & 1.819585000 & -2.477424000 \\ \mathrm{O} & 2.257600000 & 1.690377000 & 2.410750000 \\ \mathrm{H} & 1.491663000 & 1.159026000 & 2.112998000 \\ \mathrm{H} & 2.952061000 & 1.053531000 & 2.605655000 \\ \mathrm{H} & -7.995980000 & 2.306347000 & 1.699955000 \\ \mathrm{H} & -2.109929000 & 2.090616000 & 3.402173000\end{array}$

\section{$7.5\left(\mathrm{H}_{2} \mathrm{SO}_{4}\right)_{3} \cdot\left(\mathrm{NH}_{3}\right)_{3} \cdot\left(\mathrm{H}_{2} \mathrm{O}\right)_{4}$}

$\begin{array}{lrrr}\mathrm{S} & -0.639732000 & 0.029664000 & -1.011084000 \\ \mathrm{O} & -0.981082000 & -0.680288000 & 0.238708000 \\ \mathrm{O} & 0.917666000 & -0.317965000 & -1.315609000 \\ \mathrm{O} & -1.310238000 & -0.513421000 & -2.194687000\end{array}$




$\begin{array}{lrrr}\mathrm{H} & 1.454310000 & 0.136975000 & -0.650940000 \\ \mathrm{~S} & -4.045291000 & 4.518431000 & 1.176388000 \\ \mathrm{O} & -5.006237000 & 4.086852000 & 0.136517000 \\ \mathrm{O} & -3.784692000 & 6.064957000 & 0.738854000 \\ \mathrm{O} & -4.605939000 & 4.556423000 & 2.519002000 \\ \mathrm{H} & -3.180771000 & 6.451055000 & 1.388991000 \\ \mathrm{~S} & -5.399472000 & -0.282694000 & 0.824528000 \\ \mathrm{O} & -4.603595000 & -0.064356000 & -0.411728000 \\ \mathrm{O} & -5.156649000 & -1.614970000 & 1.379804000 \\ \mathrm{O} & -6.806870000 & 0.073375000 & 0.683230000 \\ \mathrm{O} & -4.855417000 & 0.789853000 & 1.884230000 \\ \mathrm{H} & -5.580556000 & 3.312759000 & 3.562820000 \\ \mathrm{O} & -0.725889000 & 1.476157000 & -0.862562000 \\ \mathrm{~N} & -3.451624000 & 2.281583000 & -1.416128000 \\ \mathrm{O} & -6.241290000 & 2.610403000 & 3.701380000 \\ \mathrm{O} & -2.341087000 & 1.189917000 & 2.008059000 \\ \mathrm{~N} & -2.881579000 & -2.755428000 & 0.350658000 \\ \mathrm{H} & -2.247490000 & 2.124863000 & 1.751559000 \\ \mathrm{H} & -3.832980000 & 0.848922000 & 1.907750000 \\ \mathrm{H} & -1.732580000 & 0.670609000 & 1.456837000 \\ \mathrm{H} & -7.021030000 & 2.755641000 & 2.232413000 \\ \mathrm{H} & -5.756039000 & 1.792535000 & 3.515556000 \\ \mathrm{H} & -2.613426000 & -3.684192000 & 0.663062000 \\ \mathrm{H} & -3.176516000 & -2.744056000 & -0.665856000 \\ \mathrm{H} & -2.090260000 & -2.088676000 & 0.425693000 \\ \mathrm{H} & -2.542424000 & 2.365711000 & -0.946033000 \\ \mathrm{H} & -4.030547000 & 3.098235000 & -1.173980000 \\ \mathrm{H} & -3.924013000 & 1.433028000 & -1.042776000 \\ \mathrm{H} & -3.262416000 & 2.153543000 & -2.429130000 \\ \mathrm{O} & -2.755482000 & 3.838113000 & 1.065597000 \\ \mathrm{H} & -1.969519000 & 0.705688000 & -3.461094000 \\ \mathrm{O} & -2.487064000 & 1.415148000 & -3.886007000 \\ \mathrm{H} & -1.852811000 & 1.916008000 & -4.407624000 \\ \mathrm{~N} & -7.266657000 & 2.758683000 & 1.200163000 \\ \mathrm{H} & -8.195688000 & 3.137335000 & 1.038724000 \\ \mathrm{H} & -6.542846000 & 3.328331000 & 0.718864000 \\ \mathrm{H} & -3.700864000 & -2.391444000 & 0.892128000 \\ \mathrm{O} & -3.660564000 & -2.147845000 & -2.160789000 \\ \mathrm{H} & -4.233798000 & -1.445036000 & -1.808460000 \\ \mathrm{H} & -2.854957000 & -1.673346000 & -2.424978000 \\ \mathrm{H} & -7.201938000 & 1.768056000 & 0.877107000\end{array}$




\section{$7.6\left(\mathrm{H}_{2} \mathrm{SO}_{4}\right)_{3} \cdot(\mathrm{DMA})_{3}$}

$\begin{array}{rrrr}\mathrm{S} & -0.744766000 & -2.011245000 & -0.512030000 \\ \mathrm{O} & 0.393703000 & -2.924461000 & -0.472328000 \\ \mathrm{O} & -1.925273000 & -2.580201000 & -1.172550000 \\ \mathrm{O} & -1.090061000 & -1.404905000 & 0.784946000 \\ \mathrm{O} & -0.239175000 & -0.796298000 & -1.453700000 \\ \mathrm{H} & 0.489993000 & 2.507112000 & -0.271332000 \\ \mathrm{~N} & 2.793517000 & -2.619062000 & 0.818621000 \\ \mathrm{H} & 1.867737000 & -2.639752000 & 0.334523000 \\ \mathrm{H} & 3.344286000 & -1.842986000 & 0.365496000 \\ \mathrm{O} & 1.980143000 & 0.344722000 & 0.464973000 \\ \mathrm{O} & 4.082472000 & -0.546135000 & -0.395618000 \\ \mathrm{~S} & 3.036094000 & 0.477673000 & -0.547683000 \\ \mathrm{O} & 3.548398000 & 1.840958000 & -0.691413000 \\ \mathrm{O} & 2.314925000 & 0.189255000 & -1.969793000 \\ \mathrm{H} & 1.493784000 & -0.321989000 & -1.807880000 \\ \mathrm{H} & -3.316131000 & -1.791551000 & -0.630588000 \\ \mathrm{H} & 2.119759000 & 2.839197000 & -0.405421000 \\ \mathrm{~N} & 1.209729000 & 3.233372000 & -0.077197000 \\ \mathrm{C} & 1.295560000 & 3.431883000 & 1.397588000 \\ \mathrm{C} & 0.843045000 & 4.453707000 & -0.840707000 \\ \mathrm{C} & 3.477680000 & -3.914348000 & 0.586583000 \\ \mathrm{C} & 2.567091000 & -2.275408000 & 2.246689000 \\ \mathrm{H} & 2.085571000 & -1.300666000 & 2.273171000 \\ \mathrm{H} & 3.526497000 & -2.234289000 & 2.762414000 \\ \mathrm{H} & 1.926486000 & -3.030449000 & 2.702173000 \\ \mathrm{H} & 2.876468000 & -4.723138000 & 1.001345000 \\ \mathrm{H} & 4.459103000 & -3.896781000 & 1.060160000 \\ \mathrm{H} & 3.591588000 & -4.058764000 & -0.486701000 \\ \mathrm{H} & -0.145676000 & 4.777326000 & -0.519497000 \\ \mathrm{H} & 1.582455000 & 5.232606000 & -0.655344000 \\ \mathrm{H} & 0.824488000 & 4.207173000 & -1.901313000 \\ \mathrm{H} & 2.062077000 & 4.177639000 & 1.608308000 \\ \mathrm{H} & 0.322852000 & 3.754233000 & 1.763436000 \\ \mathrm{H} & 1.567678000 & 2.475495000 & 1.837122000 \\ \mathrm{~S} & -2.009421000 & 1.895239000 & 0.435056000 \\ \mathrm{O} & -2.001544000 & 3.287599000 & 0.808879000 \\ \mathrm{O} & -1.018037000 & 1.580244000 & -0.641283000 \\ \mathrm{O} & -3.326918000 & 1.324636000 & 0.119772000 \\ \mathrm{H} & -0.619433000 & 0.076865000 & -1.138925000 \\ \mathrm{H} & -1.457905000 & 1.116304000 & 1.733108000 \\ \mathrm{~N} & -1.205979000 & 0.203327000 & 1.454802000 \\ & -4.107108000 & -1.235366000 & -0.221039000\end{array}$




$\begin{array}{lrrr}\mathrm{C} & -4.425913000 & -1.783155000 & 1.122596000 \\ \mathrm{C} & -5.247412000 & -1.202170000 & -1.169040000 \\ \mathrm{H} & -3.740754000 & -0.257249000 & -0.098918000 \\ \mathrm{H} & -4.795302000 & -2.803579000 & 1.020118000 \\ \mathrm{H} & -3.508734000 & -1.778661000 & 1.707859000 \\ \mathrm{H} & -5.178837000 & -1.155685000 & 1.598834000 \\ \mathrm{H} & -5.621410000 & -2.213749000 & -1.327199000 \\ \mathrm{H} & -6.037193000 & -0.570780000 & -0.762802000 \\ \mathrm{H} & -4.900988000 & -0.786988000 & -2.114221000\end{array}$

\section{$7.7\left(\mathrm{H}_{2} \mathrm{SO}_{4}\right)_{3} \cdot(\mathrm{DMA})_{3} \cdot \mathrm{H}_{2} \mathrm{O}$}

$\begin{array}{lrrr}\mathrm{S} & 3.358368000 & -0.387896000 & 0.433027000 \\ \mathrm{O} & 2.910414000 & -1.782311000 & 0.421015000 \\ \mathrm{O} & 4.933698000 & -0.502056000 & 0.674863000 \\ \mathrm{O} & 3.161070000 & 0.314326000 & -0.839705000 \\ \mathrm{H} & 5.251329000 & 0.409762000 & 0.896044000 \\ \mathrm{~S} & -2.393581000 & -1.562859000 & 0.833344000 \\ \mathrm{O} & -2.232016000 & -0.343707000 & 1.692748000 \\ \mathrm{O} & -3.655985000 & -2.229133000 & 0.987039000 \\ \mathrm{O} & -2.402024000 & -0.983287000 & -0.678867000 \\ \mathrm{~S} & -0.056879000 & 1.709958000 & -0.546894000 \\ \mathrm{O} & -0.079713000 & 0.236976000 & -0.753001000 \\ \mathrm{O} & -0.034893000 & 2.062187000 & 0.872731000 \\ \mathrm{O} & -1.137010000 & 2.389162000 & -1.276067000 \\ \mathrm{O} & 1.287598000 & 2.206396000 & -1.228183000 \\ \mathrm{H} & 1.993369000 & 1.518963000 & -1.079204000 \\ \mathrm{O} & 2.825936000 & 0.380984000 & 1.582730000 \\ \mathrm{~N} & 0.371117000 & -0.066960000 & 2.796247000 \\ \mathrm{~N} & -3.259644000 & 1.910657000 & 0.411914000 \\ \mathrm{~N} & 0.801482000 & -2.817110000 & -0.930792000 \\ \mathrm{H} & 1.618179000 & -2.332387000 & -0.488654000 \\ \mathrm{H} & -0.025838000 & -2.534783000 & -0.362495000 \\ \mathrm{H} & -2.541845000 & 2.102084000 & -0.330840000 \\ \mathrm{H} & -2.897767000 & 1.080436000 & 0.938243000 \\ \mathrm{H} & -1.542581000 & -0.486144000 & -0.807476000 \\ \mathrm{H} & 1.139759000 & 0.126458000 & 2.123633000 \\ \mathrm{H} & -0.523476000 & -0.100289000 & 2.266757000 \\ \mathrm{O} & -1.186586000 & -2.406214000 & 0.932231000 \\ \mathrm{O} & 5.093102000 & 2.112832000 & 1.416027000 \\ \mathrm{H} & 4.951245000 & 2.681978000 & 0.651549000 \\ \mathrm{H} & 4.197811000 & 1.906432000 & 1.732741000 \\ \mathrm{C} & 0.981380000 & -4.282440000 & -0.771480000\end{array}$




$\begin{array}{rrrr}\mathrm{H} & 0.113436000 & -4.799832000 & -1.178974000 \\ \mathrm{H} & 1.885803000 & -4.592790000 & -1.293710000 \\ \mathrm{H} & 1.074541000 & -4.507960000 & 0.289142000 \\ \mathrm{C} & 0.659455000 & -2.363413000 & -2.340685000 \\ \mathrm{H} & 1.544802000 & -2.665799000 & -2.899579000 \\ \mathrm{H} & -0.232037000 & -2.815989000 & -2.774678000 \\ \mathrm{H} & 0.569403000 & -1.279897000 & -2.335485000 \\ \mathrm{C} & 0.615189000 & -1.388723000 & 3.432054000 \\ \mathrm{H} & 0.542711000 & -2.153279000 & 2.664399000 \\ \mathrm{H} & 1.608154000 & -1.385930000 & 3.879000000 \\ \mathrm{H} & -0.147382000 & -1.565584000 & 4.189997000 \\ \mathrm{C} & 0.326531000 & 1.065224000 & 3.757408000 \\ \mathrm{H} & -0.509070000 & 0.920390000 & 4.442190000 \\ \mathrm{H} & 1.263633000 & 1.093681000 & 4.312580000 \\ \mathrm{H} & 0.203176000 & 1.979932000 & 3.184205000 \\ \mathrm{C} & -3.293709000 & 3.086004000 & 1.319202000 \\ \mathrm{H} & -3.949821000 & 2.874977000 & 2.163643000 \\ \mathrm{H} & -2.276309000 & 3.270457000 & 1.659097000 \\ \mathrm{H} & -3.660220000 & 3.953313000 & 0.770499000 \\ \mathrm{C} & -4.561860000 & 1.552982000 & -0.209410000 \\ \mathrm{H} & -4.406526000 & 0.675949000 & -0.834399000 \\ \mathrm{H} & -5.282793000 & 1.319296000 & 0.573681000 \\ \mathrm{H} & -4.917585000 & 2.390683000 & -0.808967000\end{array}$

\section{$7.8\left(\mathrm{H}_{2} \mathrm{SO}_{4}\right)_{3} \cdot(\mathrm{DMA})_{3} \cdot\left(\mathrm{H}_{2} \mathrm{O}\right)_{2}$}

$\begin{array}{lrrr}\mathrm{S} & -0.292508000 & -1.751065000 & -0.063166000 \\ \mathrm{O} & -1.138815000 & -1.813465000 & -1.273607000 \\ \mathrm{O} & 0.618316000 & -3.030968000 & -0.139753000 \\ \mathrm{O} & 0.598183000 & -0.580108000 & -0.043461000 \\ \mathrm{O} & -1.110299000 & -1.834570000 & 1.161183000 \\ \mathrm{H} & -0.061897000 & 1.880950000 & -1.678695000 \\ \mathrm{~N} & -3.768874000 & -1.502673000 & -0.457154000 \\ \mathrm{H} & -2.828389000 & -1.815593000 & -0.782925000 \\ \mathrm{H} & -3.570183000 & -0.580640000 & 0.007188000 \\ \mathrm{O} & -1.256973000 & 2.176800000 & 1.406801000 \\ \mathrm{O} & -3.056841000 & 0.974917000 & 0.302350000 \\ \mathrm{~S} & -2.562213000 & 1.512633000 & 1.608634000 \\ \mathrm{O} & -3.532522000 & 2.258082000 & 2.356838000 \\ \mathrm{O} & -2.255407000 & 0.201604000 & 2.505121000 \\ \mathrm{H} & -1.765147000 & -0.477805000 & 1.975709000 \\ \mathrm{H} & 1.460167000 & -2.879321000 & 0.406543000 \\ \mathrm{H} & 1.355769000 & 2.273339000 & -0.818959000\end{array}$




$\begin{array}{rrrr}\mathrm{N} & 0.702272000 & 2.593783000 & -1.569063000 \\ \mathrm{C} & 0.074600000 & 3.871008000 & -1.135968000 \\ \mathrm{C} & 1.467816000 & 2.683407000 & -2.840631000 \\ \mathrm{C} & -4.316144000 & -2.468743000 & 0.530149000 \\ \mathrm{C} & -4.634470000 & -1.239835000 & -1.635883000 \\ \mathrm{H} & -4.126577000 & -0.520309000 & -2.276660000 \\ \mathrm{H} & -5.583727000 & -0.824103000 & -1.299690000 \\ \mathrm{H} & -4.803650000 & -2.168769000 & -2.180062000 \\ \mathrm{H} & -4.513596000 & -3.419313000 & 0.034605000 \\ \mathrm{H} & -5.238256000 & -2.068812000 & 0.951090000 \\ \mathrm{H} & -3.572380000 & -2.605313000 & 1.312203000 \\ \mathrm{H} & 0.786523000 & 2.937723000 & -3.652110000 \\ \mathrm{H} & 2.240044000 & 3.445629000 & -2.741116000 \\ \mathrm{H} & 1.939869000 & 1.720223000 & -3.023936000 \\ \mathrm{H} & 0.855960000 & 4.606698000 & -0.946062000 \\ \mathrm{H} & -0.589331000 & 4.227713000 & -1.923445000 \\ \mathrm{H} & -0.496550000 & 3.675940000 & -0.228893000 \\ \mathrm{~S} & 3.587217000 & 0.926075000 & 0.028710000 \\ \mathrm{O} & 4.808676000 & 1.456404000 & -0.502306000 \\ \mathrm{O} & 2.561154000 & 1.970829000 & 0.372789000 \\ \mathrm{O} & 3.696224000 & -0.022637000 & 1.148384000 \\ \mathrm{H} & 1.723022000 & 1.485343000 & 1.808403000 \\ \mathrm{O} & 2.912533000 & 0.109725000 & -1.200168000 \\ \mathrm{H} & 2.068886000 & -0.280530000 & -0.863958000 \\ \mathrm{~N} & 1.088185000 & 1.446692000 & 2.638198000 \\ \mathrm{H} & 0.134322000 & 1.602401000 & 2.243116000 \\ \mathrm{O} & -1.487183000 & 0.975306000 & -2.009045000 \\ \mathrm{H} & -2.034933000 & 1.112737000 & -1.209135000 \\ \mathrm{H} & -1.245385000 & 0.034478000 & -1.961614000 \\ \mathrm{O} & 2.756695000 & -2.560650000 & 1.255275000 \\ \mathrm{H} & 3.114536000 & -1.646358000 & 1.168423000 \\ \mathrm{H} & 3.509769000 & -3.150866000 & 1.160551000 \\ \mathrm{C} & 1.171093000 & 0.117417000 & 3.300827000 \\ \mathrm{H} & 0.887196000 & -0.644191000 & 2.580733000 \\ \mathrm{H} & 2.196453000 & -0.052181000 & 3.624155000 \\ \mathrm{H} & 0.486626000 & 0.100793000 & 4.148488000 \\ \mathrm{C} & 1.420700000 & 2.590538000 & 3.524299000 \\ \mathrm{H} & 0.720685000 & 2.617876000 & 4.358808000 \\ \mathrm{H} & 2.440113000 & 2.475217000 & 3.890935000 \\ \mathrm{H} & 1.340946000 & 3.511295000 & 2.949435000\end{array}$

\section{$7.9\left(\mathrm{H}_{2} \mathrm{SO}_{4}\right)_{3} \cdot(\mathrm{DMA})_{3} \cdot\left(\mathrm{H}_{2} \mathrm{O}\right)_{3}$}




$\begin{array}{lrrr}\mathrm{S} & -0.027194000 & -0.324670000 & 0.668877000 \\ \mathrm{O} & -0.003874000 & 0.553709000 & 1.865087000 \\ \mathrm{O} & 1.370778000 & -0.165527000 & -0.043460000 \\ \mathrm{O} & -0.125707000 & -1.745243000 & 1.024540000 \\ \mathrm{H} & 1.380854000 & 0.728996000 & -0.525443000 \\ \mathrm{~S} & -3.927511000 & 4.035873000 & 1.592321000 \\ \mathrm{O} & -4.363025000 & 3.681326000 & 0.237488000 \\ \mathrm{O} & -4.094421000 & 5.659334000 & 1.706839000 \\ \mathrm{O} & -4.835739000 & 3.576503000 & 2.645468000 \\ \mathrm{H} & -3.487931000 & 6.057823000 & 1.067345000 \\ \mathrm{~S} & -4.945266000 & -0.623640000 & 1.086186000 \\ \mathrm{O} & -4.712033000 & -0.456987000 & -0.466759000 \\ \mathrm{O} & -4.308740000 & -1.901754000 & 1.449048000 \\ \mathrm{O} & -6.397380000 & -0.609492000 & 1.308940000 \\ \mathrm{O} & -4.306510000 & 0.543098000 & 1.734395000 \\ \mathrm{H} & -6.083737000 & 2.507549000 & 2.135639000 \\ \mathrm{O} & -1.059696000 & 0.082444000 & -0.297439000 \\ \mathrm{O} & -3.261412000 & 1.501464000 & -1.072300000 \\ \mathrm{~N} & -6.982491000 & 2.035136000 & 1.880587000 \\ \mathrm{~N} & -2.218939000 & 0.597349000 & 3.470352000 \\ \mathrm{~N} & -2.321512000 & -2.900802000 & -0.162362000 \\ \mathrm{H} & -3.039511000 & 0.663159000 & 2.824918000 \\ \mathrm{H} & -1.375696000 & 0.619105000 & 2.850143000 \\ \mathrm{H} & -6.764651000 & 1.026676000 & 1.696866000 \\ \mathrm{H} & -1.449956000 & -2.469670000 & 0.229765000 \\ \mathrm{H} & -3.112871000 & -2.451628000 & 0.357357000 \\ \mathrm{H} & -2.405212000 & 1.245339000 & -0.683081000 \\ \mathrm{H} & -3.556770000 & 2.320182000 & -0.625334000 \\ \mathrm{H} & -4.140724000 & 0.387958000 & -0.693294000 \\ \mathrm{O} & -2.515915000 & 3.749424000 & 1.846028000 \\ \mathrm{O} & 1.152517000 & 2.151203000 & -1.232610000 \\ \mathrm{H} & 0.383319000 & 2.014391000 & -1.796598000 \\ \mathrm{H} & 0.824742000 & 2.733488000 & -0.503208000 \\ \mathrm{O} & 0.195885000 & 3.301944000 & 1.015931000 \\ \mathrm{H} & 0.247723000 & 2.461342000 & 1.499117000 \\ \mathrm{H} & -0.724479000 & 3.584496000 & 1.137737000 \\ \mathrm{C} & -2.268447000 & -0.728956000 & 4.138092000 \\ \mathrm{H} & -3.125921000 & -0.760471000 & 4.809944000 \\ \mathrm{H} & -2.381756000 & -1.494778000 & 3.374116000 \\ \mathrm{H} & -1.345844000 & -0.886801000 & 4.695529000 \\ \mathrm{H} & -2.188489000 & 1.759840000 & 4.397178000 \\ \mathrm{H} & -3.089925000 & 1.751310000 & 5.009338000 \\ & -1.304968000 & 1.686239000 & 5.031739000 \\ -2.161716000 & 2.669548000 & 3.800910000\end{array}$




$\begin{array}{ll}\mathrm{C} & -7.912462000 \\ \mathrm{H} & -8.857362000 \\ \mathrm{H} & -7.463525000 \\ \mathrm{H} & -8.077165000 \\ \mathrm{C} & -7.493770000 \\ \mathrm{H} & -6.704481000 \\ \mathrm{H} & -8.373379000 \\ \mathrm{H} & -7.744576000 \\ \mathrm{C} & -2.419114000 \\ \mathrm{H} & -1.605028000 \\ \mathrm{H} & -3.382059000 \\ \mathrm{H} & -2.329920000 \\ \mathrm{C} & -2.329520000 \\ \mathrm{H} & -3.256922000 \\ \mathrm{H} & -1.474451000 \\ \mathrm{H} & -2.264675000\end{array}$

$\begin{array}{rr}2.134024000 & 3.031802000 \\ 1.653242000 & 2.779299000 \\ 1.630167000 & 3.886370000 \\ 3.183721000 & 3.272602000 \\ 2.647990000 & 0.625292000 \\ 2.602136000 & -0.121021000 \\ 2.098953000 & 0.290280000 \\ 3.691366000 & 0.813376000 \\ -2.585789000 & -1.613308000 \\ -3.084973000 & -2.139374000 \\ -2.926835000 & -1.990730000 \\ -1.509501000 & -1.729629000 \\ -4.351043000 & 0.152762000 \\ -4.794296000 & -0.208818000 \\ -4.830696000 & -0.322953000 \\ -4.473911000 & 1.232647000\end{array}$

\section{$7.10\left(\mathrm{H}_{2} \mathrm{SO}_{4}\right)_{3} \cdot(\mathrm{DMA})_{3} \cdot\left(\mathrm{H}_{2} \mathrm{O}\right)_{4}$}

$\begin{array}{lrrr}\mathrm{S} & -0.160074000 & -0.314741000 & -0.172176000 \\ \mathrm{O} & -0.660300000 & 0.756755000 & 0.706870000 \\ \mathrm{O} & 1.273068000 & -0.532793000 & -0.070563000 \\ \mathrm{O} & -0.966043000 & -1.550171000 & -0.043593000 \\ \mathrm{H} & 2.019249000 & 1.021043000 & 0.778039000 \\ \mathrm{~S} & -2.302034000 & 3.831764000 & 1.052107000 \\ \mathrm{O} & -1.920266000 & 3.692589000 & -0.374631000 \\ \mathrm{O} & -3.414389000 & 4.950223000 & 1.133218000 \\ \mathrm{O} & -2.921224000 & 2.604044000 & 1.590496000 \\ \mathrm{H} & -4.312597000 & 4.530129000 & 0.958785000 \\ \mathrm{~S} & -5.216234000 & 0.012340000 & 0.873970000 \\ \mathrm{O} & -3.916319000 & 0.690962000 & 0.137978000 \\ \mathrm{O} & -5.526413000 & -1.073648000 & -0.062919000 \\ \mathrm{O} & -6.230117000 & 1.050420000 & 0.968774000 \\ \mathrm{O} & -4.706653000 & -0.458327000 & 2.169066000 \\ \mathrm{H} & -3.538731000 & 1.435927000 & 0.692485000 \\ \mathrm{O} & -0.383624000 & 0.210987000 & -1.677544000 \\ \mathrm{O} & -2.600375000 & 1.575765000 & -2.078215000 \\ \mathrm{~N} & -2.119583000 & 0.049527000 & 2.994693000 \\ \mathrm{~N} & -3.319058000 & -2.187858000 & -1.237881000 \\ \mathrm{H} & -3.084365000 & -0.167144000 & 2.660157000 \\ \mathrm{H} & -1.635272000 & 0.507584000 & 2.195009000 \\ \mathrm{H} & -2.456701000 & -1.819765000 & -0.763737000 \\ \mathrm{H} & -4.149124000 & -1.703823000 & -0.814129000\end{array}$




$\begin{array}{lrrr}\mathrm{H} & -1.254131000 & 0.709871000 & -1.765393000 \\ \mathrm{H} & -2.419229000 & 2.441704000 & -1.665124000 \\ \mathrm{H} & -3.287110000 & 1.212905000 & -1.490674000 \\ \mathrm{O} & -1.186262000 & 4.319223000 & 1.860209000 \\ \mathrm{~N} & 0.894276000 & 3.564302000 & -0.632234000 \\ \mathrm{H} & -0.135592000 & 3.480153000 & -0.522201000 \\ \mathrm{O} & 2.179745000 & 1.931297000 & 1.091368000 \\ \mathrm{H} & 1.349913000 & 2.890101000 & 0.054947000 \\ \mathrm{H} & 1.657379000 & 2.022932000 & 1.913484000 \\ \mathrm{O} & 0.529664000 & 2.629087000 & 3.252924000 \\ \mathrm{H} & -0.138087000 & 3.183249000 & 2.796867000 \\ \mathrm{H} & 0.926333000 & 3.208427000 & 3.910842000 \\ \mathrm{O} & -5.734708000 & 3.799450000 & 0.633927000 \\ \mathrm{H} & -5.896266000 & 2.874616000 & 0.907867000 \\ \mathrm{H} & -6.070496000 & 3.853059000 & -0.265615000 \\ \mathrm{C} & -2.214949000 & 0.980557000 & 4.153200000 \\ \mathrm{H} & -2.662146000 & 0.450614000 & 4.994039000 \\ \mathrm{H} & -1.215139000 & 1.336720000 & 4.395068000 \\ \mathrm{H} & -2.837772000 & 1.819992000 & 3.856176000 \\ \mathrm{C} & -1.404258000 & -1.219630000 & 3.291609000 \\ \mathrm{H} & -1.961841000 & -1.776326000 & 4.044456000 \\ \mathrm{H} & -1.322312000 & -1.792041000 & 2.369570000 \\ \mathrm{H} & -0.406929000 & -0.984707000 & 3.661841000 \\ \mathrm{C} & -3.423670000 & -3.637230000 & -0.930688000 \\ \mathrm{H} & -4.310811000 & -4.047050000 & -1.412753000 \\ \mathrm{H} & -2.528707000 & -4.146193000 & -1.286703000 \\ \mathrm{H} & -3.510183000 & -3.755838000 & 0.147902000 \\ \mathrm{C} & -3.237011000 & -1.880093000 & -2.690293000 \\ \mathrm{H} & -2.347525000 & -2.352839000 & -3.105380000 \\ \mathrm{H} & -4.131374000 & -2.253888000 & -3.188307000 \\ \mathrm{H} & -3.163874000 & -0.802363000 & -2.822819000 \\ \mathrm{C} & 1.285451000 & 3.170145000 & -2.011384000 \\ \mathrm{H} & 2.368928000 & 3.241763000 & -2.106231000 \\ \mathrm{H} & 0.966236000 & 2.143082000 & -2.182936000 \\ \mathrm{H} & 0.806197000 & 3.837359000 & -2.728312000 \\ \mathrm{C} & 1.282048000 & 4.949845000 & -0.260060000 \\ \mathrm{H} & 0.882094000 & 5.157099000 & 0.730118000 \\ \mathrm{H} & 2.369001000 & 5.028654000 & -0.261672000 \\ \mathrm{H} & 0.852824000 & 5.650863000 & -0.975800000\end{array}$

